\title{
HORIZON-SCALE LEPTON ACCELERATION IN JETS: EXPLAINING THE COMPACT RADIO EMISSION IN M87
}

\author{
AVEry E. BRoderick, ${ }^{1,2}$ AND AlEXANDER TCHEKHOVSKOY ${ }^{3,4}$ \\ ${ }^{1}$ Perimeter Institute for Theoretical Physics, 31 Caroline Street North, Waterloo, ON, N2L 2Y5, Canada \\ 2 Department of Physics and Astronomy, University of Waterloo, 200 University Avenue West, Waterloo, ON, N2L 3G1, Canada \\ ${ }^{3}$ Departments of Astronomy and Physics, University of California Berkeley, Berkeley, CA 94720-3411, USA; Einstein Fellow \\ ${ }^{4}$ Lawrence Berkeley National Laboratory, 1 Cyclotron Rd, Berkeley, CA 94720, USA \\ Draft version September 16, 2018
}

\begin{abstract}
It has now become clear that the radio jet in the giant elliptical galaxy M87 must turn on very close to the black hole. This implies the efficient acceleration of leptons within the jet at scales much smaller than feasible by the typical dissipative events usually invoked to explain jet synchrotron emission. Here we show that the stagnation surface, the separatrix between material that falls back into the black hole and material that is accelerated outward forming the jet, is a natural site of pair formation and particle acceleration. This occurs via an inverse-Compton pair catastrophe driven by unscreened electric fields within the charge-starved region about the stagnation surface and substantially amplified by a post-gap cascade. For typical estimates of the jet properties in M87, we find excellent quantitive agreement between the predicted relativistic lepton densities and those required by recent highfrequency radio observations of M87. This mechanism fails to adequately fill a putative jet from Sagittarius A* with relativistic leptons, which may explain the lack of an obvious radio jet in the Galactic center. Finally, this process implies a relationship between the kinetic jet power and the gamma-ray luminosity of blazars, produced during the post-gap cascade.

Subject headings: accretion, accretion disks - black hole physics - galaxies: individual (M87) - galaxies: jets gamma rays: galaxies - radio continuum: galaxies
\end{abstract}

\section{INTRODUCTION}

Roughly $10 \%$ of active galactic nuclei (AGN), exhibit powerful radio jets. These can extend for intergalactic distances, and potentially have impacts for the formation and evolution of their galactic environments. Great strides have been made in the past two decades in the theoretical modeling of the structure and formation of AGN jets, with current general relativistic magnetohydrodynamic (GRMHD) models now capable of reproducing the structure and Lorentz factors $(\Gamma)$ of observed objects. In these, large-scale electromagnetic fields sourced and collimated by the surrounding accretion flow efficiently extract the rotational energy of the black hole (Blandford \& Znajek 1977; Tchekhovskoy et al. 2010, 2011, 2012; Tchekhovskoy \& McKinney 2012; McKinney et al. 2012; Tchekhovskoy 2015). Within the context of GRMHD simulations, a canonical outflow structure has emerged, consisting of a central relativistic jet core surrounded by a moderately relativistic jet sheath, followed by a non-relativistic disk wind.

Less certain is why jets shine. This has become especially pressing now that it has become clear that the jet in M87 exhibits horizon-scale structure, implying emission on horizon scales (Doeleman et al. 2012). It is widely believed that from the radio to the UV, the observed jet emission is due to synchrotron emission from a population of nonthermal leptons. Not presently known is the mechanism by which this population is produced. Uncertain are the processes by which jets are mass loaded, particles within the jet are accelerated, and the dynamical structures associated with the observed jet at different wavelengths (e.g., the jet spine vs. jet sheath). Any candidate mechanisms must simultaneously explain two critical features.

First, the leptons must be located within the fast region of the jet. Radio observations far from the radio core show superluminal motions which imply large values of the bulk Lorentz factor, $\Gamma \gg 1$. Thus the synchrotron emitting particles in par- ticular must be moving at these extreme velocities. This suggests that the radio emission is not due to a mass-loaded, transrelativistic disk wind surrounding and potentially collimating the jet. This is problematic because jet formation simulations, as well as approximate analytical models, imply the presence of large-scale, dynamically dominant, ordered magnetic fields. Correspondingly, the exchange of particles onto the jet magnetic field lines is dramatically suppressed, with the consequence that this is unlikely to be the mechanism by which jets are mass loaded. Funnel regions can potentially be filled with pairs via collisions of gamma-rays formed in the disk. These gamma-rays are presumably produced through a sequence of inverse Compton scatterings of disk sub-mm photons with the hot disk electrons (Levinson \& Rieger 2011; Mościbrodzka et al. 2011), though such a mechanism requires order unity radiative efficiency of the accretion flow. However, for the radiatively inefficient accretion flows believed to be relevant at the low Eddington ratio observed in objects like M87, most of the sub-mm emission is produced in the jet, and this process is suppressed. Alternative processes, such as neutron diffusion (Levinson \& Eichler 2003), have been suggested as a means to accomplish this, though fail at low source luminosities (e.g., M87).

Second, the leptons must be accelerated. Simply mass loading a jet is insufficient; assuming the primary emission mechanism is synchrotron emission, the leptons must also be accelerated to large internal Lorentz factors as well. Far from the black hole this may be accomplished via internal shocks, magnetic reconnection, damping of plasma waves, etc. However, already within $10 r_{\mathrm{g}}$ bright radio emission is detected in M87, where $r_{\mathrm{g}} \equiv G M / c^{2}$ is the black hole's gravitational radius (Doeleman et al. 2012). Thus, particle acceleration must also be occurring quite close to the black hole, where these processes either do not occur or are strongly suppressed.

Within the context of M87, there are numerous spectral and 
Table 1

Key Symbol Definitions

\begin{tabular}{rcll}
\hline \hline Name & Eq. & Definition \\
\hline$u_{s}$ & 5 & Density of seed photons \\
$n_{g}$ & 18 & Number density of charges in the gap \\
$n_{\infty}$ & 28 & Number density of charges at the end of the cascade \\
$\ell_{\mathrm{IC}}$ & 6 & Cooling length of electrons due to inverse Compton scattering off seed photons \\
$\ell_{\gamma \gamma}$ & 15 & Mean free path of $\gamma$-ray photons due to pair production on seed photons \\
$\ell_{\mathrm{C}}$ & 10 & Cooling length of electrons due to curvature radiation \\
$\Delta$ & 17 & Gap thickness \\
$\gamma_{\max , \mathrm{IC}}$ & 8 & Maximum electron Lorentz factor due to the acceleration over the distance $\ell_{\mathrm{IC}}$ \\
$\gamma_{\max , \mathrm{C}}$ & 11 & Maximum electron Lorentz factor due to the acceleration over the distance $\ell_{\mathrm{C}}$ \\
$\gamma_{\max }$ & 3 & Maximum electron Lorentz factor due to the acceleration over the distance $\Delta$ \\
$\gamma_{\gamma \gamma}$ & 27 & Lorentz factor of electrons below which they do not encounter enough seed photons to pair produce \\
$\gamma_{\infty}$ & 29 & Lorentz factor of electrons below which they no longer efficiently Compton cool \\
$\Omega_{\mathrm{F}}$ & 2 & Field line angular rotation frequency \\
$B$ & 44 & Magnetic field strength at the stagnation surface, $r \sim 10 r_{g}$ \\
\hline \hline
\end{tabular}

morphological constraints that should inform any attempt to model the lepton loading and acceleration in jets from low luminosity AGN (LLAGN; potentially comprising the majority of the population). Most importantly, at a wavelength of $7 \mathrm{~mm}$ and shorter the luminosity from the extended jet considerably exceeds that from the core, and thus importantly the luminosity is not core dominated. This morphology is inconsistent with the core-dominated morphology produced by models in which the bulk of the accelerated leptons are produced at the black hole, e.g., models in which nonthermal energy in electron-positron pairs is $u_{e^{ \pm}} \propto B^{2}$ (Mościbrodzka et al. 2009; Shcherbakov et al. 2012; Dexter et al. 2012; Mościbrodzka $\&$ Falcke 2013) or with the annihilation of high-energy photons produced in low-luminosity radiatively-inefficient accretion flows (Mościbrodzka et al. 2011). It is, however, consistent with models in which the energetic lepton population is generated at a height of roughly $10 r_{\mathrm{g}}$ (Broderick \& Loeb 2009).

This height is suspiciously similar to the location of the stagnation surface: the location at which the electromagnetically driven outflow is arrested by the gravity of the black hole. At present, the stagnation surface is not consistently treated in simulations, which often assume a sufficient number of charged particles to maintain the electromagnetic currents required by the electrodynamics. However, the evacuation of this region (above by outflow, below by accretion) should result in the creation of large electric fields, potentially capable of both loading the jet with leptons and accelerating these leptons to high energies.

Acceleration of particles at gaps in black hole magnetosphere, in analogy with particle acceleration in pulsar magnetosphere gaps, have been discussed by a number of authors (see, e.g. Beskin et al. 1992; Hirotani \& Okamoto 1998; Levinson \& Rieger 2011), including due to field-spin misalignment (Neronov \& Aharonian 2007). However, such studies are often either focused upon regions unassociated with the stagnation surface, require energetic backgrounds, or ignore the post-gap evolution of the resulting energetic particle population (Levinson \& Rieger 2011), which we find critical to reproducing the observed particle densities in M87. Existing efforts to numerically model the post-gap process have not yet self-consistently modeled the gap itself (Vincent \& Lebohec 2010). Thus, these are not immediately applicable beyond the immediate scope of their derivation, and none are directly relevant for the relativistic lepton loading of M87.

Here we show that for M87 this is indeed expected to be the case. Unscreened electric fields are capable of initiating an inverse-Compton pair-creating cascade. Within this process the number density of nonthermal particles is a function primarily of the soft-photon background produced by the surrounding accretion flow and within the jet itself. Most importantly, we show that for M87 the inferred nonthermal lepton number densities match those implied by recent mm-VLBI observations that resolve the jet launching region. In $§ 2$ we discuss physical processes responsible for particle acceleration and cooling in the gap near the stagnation surface. In $\S 3$, we discuss the properties of post-gap cascade and describe our numerical method. In $\S 4$ and $\S 5$ we discuss our results for M87 and Sgr A*, respectively. In $\$ 7$ we conclude.

\section{PARTICLE CREATION AND ACCELERATION IN THE STAGNATION SURFACE}

The stagnation surface is continually being evacuated as material either accelerates outward in the jet or inward into the black hole. In the absence of some mechanism to continually replenish the density of charges the result is a charge-starved region, incapable of screening the large electric fields that will be present. The typical electric field strength within the stagnation surface is set by the angular velocity of the magnetic field line footprints, $\Omega_{F}$, dragged about by the spinning black hole or the surrounding accretion flow,

$$
E \approx \Omega_{F} R B / c,
$$

where $R$ is the cylindrical radius of the stagnation surface. For black hole driven jets $\Omega_{F}$ is set, in turn, by the angular velocity of the black hole horizon,

$$
\Omega_{\mathrm{H}}=a c / 2 r_{\mathrm{H}},
$$

where $r_{\mathrm{H}} \equiv r_{\mathrm{g}}\left(1+\sqrt{1-a^{2}}\right)$ is the size of the horizon in BoyerLindquist coordinates. Typically, analytical studies and GRMGHD simulations find $\Omega_{F} \approx 0.25 \Omega_{\mathrm{H}}-0.5 \Omega_{\mathrm{H}}$ (Blandford \& 
Znajek 1977; Komissarov 2004; Tchekhovskoy et al. 2010; McKinney et al. 2012; Penna et al. 2013; Lasota et al. 2014).

This unscreened electric field will necessarily accelerate electrons across the charge-starved gap. That is, given a gap thickness of $\Delta$, in principle, the maximum electron Lorentz factor is

$$
\gamma_{\max } \approx e E \Delta .
$$

For gap sizes of order the gravitational radius, in the case of M87 this produces Lorentz factors of $10^{13}$, more than sufficient to produce an inverse-Compton cascade. In practice, $\gamma_{\max }$ is limited by radiative losses and the size of the gap is set by the cascade. Here we estimate the gap thickness and corresponding pair densities injected into the jet by the gap.

\subsection{Radiative Losses \\ 2.1.1. Inverse Compton}

The source of seed photons for inverse-Compton scattering is not completely clear. Nevertheless, we might assume that these are related to the observed emission from the source. Thus, if the observed luminosity is synonymous with the seed photon luminosity we will have

$$
L_{s} \approx \Omega r_{s}^{2} u_{s} c / 3=\Omega D^{2} F_{s},
$$

where $r_{s}$ is the size of the emission region, $\Omega$ is the solid angle of the emission region (i.e., beaming factor), $u_{s}$ is the local energy density of seed photons, $D$ is the source distance, and $F_{S}$ is the observed seed-photon flux. Thus, in terms of the observed flux,

$$
u_{s}=3 \frac{D^{2} F_{s}}{r_{s}^{2} c}=\frac{3 L_{s}}{4 \pi r_{s}^{2} c},
$$

where $L_{s}$ is the seed photon luminosity. The cooling length to inverse-Compton scattering is related to $u_{s}$ and $\gamma_{\max }$ via

$$
\ell_{\mathrm{IC}}=\frac{3 m_{e} c^{2}}{4 \sigma_{T} u_{s} \gamma_{\max }}
$$

where $\sigma_{T}$ is the Thomson cross section ${ }^{1}$. This defines the typical distance over which particles can accelerate within the gap, thus

$$
\gamma_{\mathrm{max}, \mathrm{IC}} m_{e} c^{2} \approx e E \ell_{\mathrm{IC}},
$$

which implies a limiting Lorentz factor of

$$
\gamma_{\max , \mathrm{IC}}=\left(\frac{3 e R \Omega_{F} B}{4 \sigma_{T} u_{S} C}\right)^{1 / 2}=4.2 \times 10^{8} R_{15}^{1 / 2} \Omega_{F,-4}^{1 / 2} B_{2}^{1 / 2} u_{s, 0}^{-1 / 2},
$$

after inserting the expression for $E$ in which we have adopted the standard notation, $R \equiv R_{15} 10^{15} \mathrm{~cm}, \Omega_{F} \equiv \Omega_{F,-4} 10^{-4} \mathrm{~s}^{-1}$, $B \equiv B_{2} 10^{2} \mathrm{G}$, and $u_{s} \equiv u_{s, 0} \mathrm{erg} \mathrm{cm}^{-3}$. The associated cooling length is $\ell_{\mathrm{IC}}=2.2 \times 10^{9} R_{15}^{-1 / 2} \Omega_{F,-4}^{-1 / 2} B_{2}^{-1 / 2} u_{s, 0}^{-1 / 2} \mathrm{~cm}$.

\subsubsection{Curvature}

Curvature radiation losses provide a second, seed-photon independent, limit upon $\gamma_{\max }$. The power emitted depends upon the curvature radius of the magnetic field, $r_{c} \approx R$, and is given by (Levinson \& Rieger 2011)

$$
P_{\mathrm{C}}=\frac{2 e^{2} c \gamma_{\max }^{4}}{3 R^{2}}
$$

${ }^{1}$ This assumes that $2 \gamma_{\max }^{2} \epsilon_{s} \lesssim \gamma_{\max } m_{e} c^{2}$, which we will verify is approximately true for M87 in what follows. If this is not true, $\ell_{\mathrm{IC}}$ is larger, producing a weaker limit upon $\gamma_{\max }$.

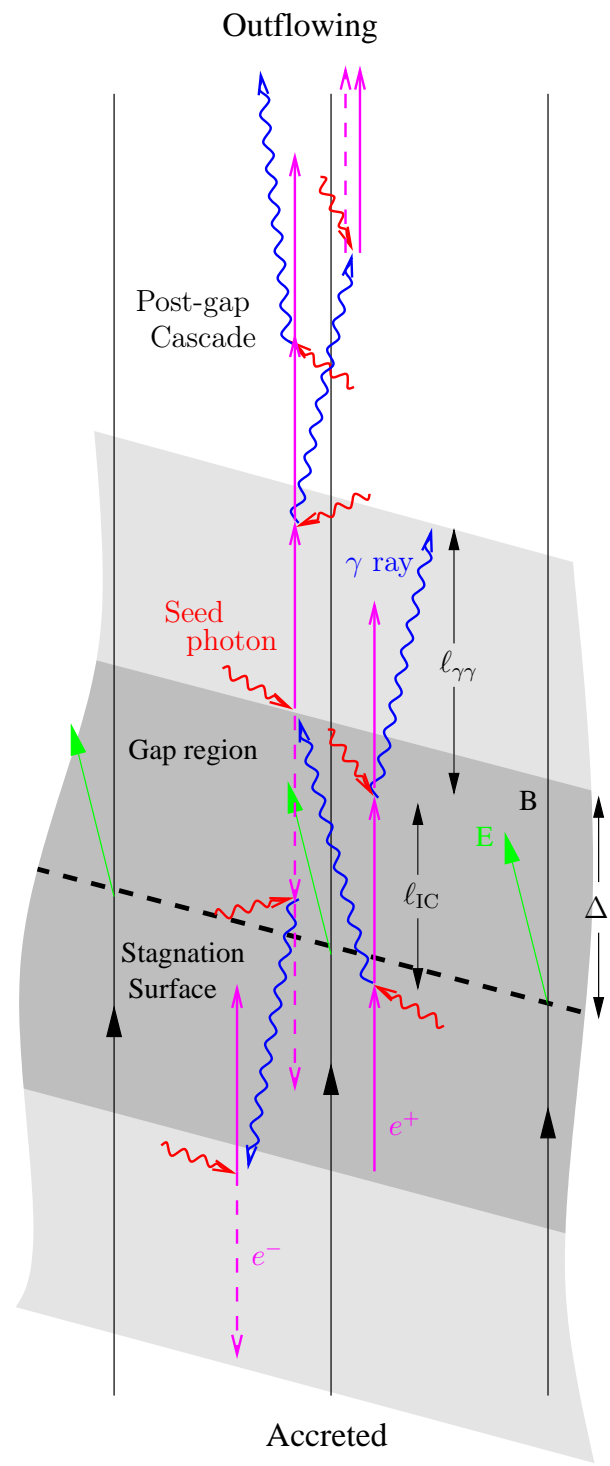

Figure 1. Schematic of a small portion of the stagnation surface and the associated gap region. Within the gap particles are rapidly accelerated to asymptotic Lorentz factors, limited by inverse Compton cooling on the ambient softphoton background (red photons), inducing a net charge separation. The resulting up-scattered gamma rays (blue photons) pair produce on the soft-photon background, resulting in a pair catastrophe. When the density in Equation (18) is reached, particle acceleration ceases, and the remaining post-gap cascade produces an asymptotic lepton density at fixed charge density. Particles propagating downward are accreted by the black hole, while those propagating upward populate the black hole jet. Due to the high Lorentz factors typically involved, the entire process is essentially one-dimensional; the oblique propagation angles of the resulting gamma rays are exaggerated for clarity. Relevant locations and characteristic length scales are labeled (see the main text for details).

The corresponding cooling length is

$$
\ell_{\mathrm{C}}=\frac{3 R^{2} m_{e} c^{2}}{2 e^{2} \gamma_{\max }^{3}}
$$

implying a limit of

$$
\gamma_{\max , \mathrm{C}}=\left(\frac{3 R^{3} \Omega_{F} B}{2 e c}\right)^{1 / 4}=3.2 \times 10^{10} R_{15}^{3 / 4} \Omega_{F,-4}^{1 / 4} B_{2}^{1 / 4} .
$$


In practice, the maximum particle Lorentz factor is set by the minimum radiative loss limit. As we will see in Section 4 the limit arising due to inverse-Compton losses is typically more severe. This is true if

$$
\begin{aligned}
u_{s}> & \left(\frac{3 e^{3} \Omega_{F} B}{8 \sigma_{T}^{2} R c}\right)^{1 / 2}=1.8 \times 10^{-4} \Omega_{F,-4}^{1 / 2} B_{2}^{1 / 2} R_{15}^{-1 / 2} \mathrm{erg} \mathrm{cm}^{-3} \\
& \Rightarrow \quad F_{s} \gtrsim 1.8 r_{s, 16}^{2} D_{26}^{-2} \Omega_{F,-4}^{1 / 2} B_{2}^{1 / 2} R_{15}^{-1 / 2} \mathrm{mJy} \mathrm{THz},
\end{aligned}
$$

where $r_{s} \equiv r_{s, 16} 10^{16} \mathrm{~cm}$ and $D \equiv D_{26} 10^{26} \mathrm{~cm}$. That is, as long as the seed photon density is sufficiently high, the gap dynamics is set by inverse-Compton losses. Even for the highly subEddington sources (e.g., M87 and Sgr A*) this is often satisfied.

\subsubsection{Synchrotron}

Generally the electric field in the gap will not be aligned with the magnetic field. As a consequence, accelerated particles will gyrate, and thus emit synchrotron radiation. This can provide an alternative source of hard photons oriented at substantial angles to the magnetic field. The cooling length for synchrotron is $\ell_{\mathrm{S}}=\ell_{\mathrm{IC}} u_{S} / u_{B}$, where $u_{B}=B^{2} / 8 \pi$, and thus we generally have $\ell_{\mathrm{S}} \ll \ell_{\mathrm{IC}}$, since the seed photon energy density is typically much smaller than the magnetic energy density in the sources of interest. However, note that synchrotron is only capable of cooling the transverse motion of the particles. While this serves to ensure in extremely short time that the leptons are traveling along magnetic field lines (making the particle acceleration essentially a one-dimensional process), it does not otherwise limit the particle Lorentz factors.

\subsubsection{Collective Processes}

Finally, we consider the importance of collective effects due to the counter-streaming electron-positron plasmas. Due to their opposite charges, electrons and positrons will be accelerated in opposite directions, producing a pair of cold, counterpropagating lepton beams. This is unstable to the well-known two-stream instability, modified slightly due to the opposite charges in the two beams (which we refer to as the "counterstreaming instability" and presented briefly in Appendix A). The instability growth rate is generally dependent upon the stream densities, corresponding to a cooling length of

$$
\ell_{\mathrm{CSI}} \approx\left(\frac{2 m_{e} \gamma_{\max }^{3}}{\pi e^{2} n_{\mathrm{g}}}\right)^{1 / 2} c
$$

and thus we cannot fully evaluate the importance of plasma instabilities without an estimate of the pair densities. However, for the estimate presented below (Equation 18 ), $\ell_{\mathrm{CSI}} \approx 7.1 \times$ $10^{18} u_{s, 0}^{-1} \eta_{\mathrm{th}}^{1 / 4} \mathrm{~cm}$, and therefore this is never relevant.

\subsection{Pair Density within the Gap}

As the limiting radiative process, inverse-Compton cooling also provides a limit upon the gap thickness. The up-scattered photons have energies of roughly $\min \left(2 \gamma_{\max }^{2} \epsilon_{s}, \gamma_{\max } m_{e} c^{2}\right)$. Thus, at meV energies, comparable to the photon energy near the peak of the spectral energy distribution (SED) of the sources of primary interest here (see Sections 4.1.4 and 5.1.3), the up-scattered photon energy is similar to that of the incident electron. Thus, the up-scattered photons will pair produce off of photons with energies above

$$
\epsilon_{\mathrm{th}} \approx \frac{4 m_{e} c^{2}}{\gamma_{\max }}=4.8 R_{15}^{-1 / 2} \Omega_{F,-4}^{-1 / 2} B_{2}^{-1 / 2} u_{s, 0}^{1 / 2} \mathrm{meV}
$$

and thus just above the SED peak for our fiducial parameters. Hence, the inverse-Compton cooling of the gap-accelerated pairs initiates a pair-production catastrophe.

The pair-production cross section peaks near $2 \epsilon_{\text {th }}$ at $\sigma_{\gamma \gamma} \approx$ $0.35 \sigma_{T}$ (Gould \& Schréder 1967). Thus, the mean free path of the up-scattered seed photons is approximately

$$
\ell_{\gamma \gamma} \approx \frac{2 \epsilon_{\mathrm{th}}}{u_{s, \mathrm{th}} \sigma_{\gamma \gamma}} \approx 6.6 \times 10^{10} R_{15}^{-1 / 2} \Omega_{F,-4}^{-1 / 2} B_{2}^{-1 / 2} u_{s, 0}^{-1 / 2} \eta_{\mathrm{th}} \mathrm{cm},
$$

where $u_{s, \text { th }} \equiv u_{s, 0} / \eta_{\text {th }}$ is the energy density of seed photons with energies above $\epsilon_{\text {th }}$. For a source with infrared spectral index $\alpha$ (i.e., $F_{\nu} \propto \nu^{-\alpha}$, in M87 $\alpha \approx 1.2$ ), the number of seed photons above an energy $\epsilon_{s}$ is $\propto \epsilon_{s}^{-\alpha}$. Thus, if there is a spectral break at $\epsilon_{b}$, below which the seed photon energy density may be neglected,

$$
\eta_{\mathrm{th}}= \begin{cases}1 & \epsilon_{\mathrm{th}}<\epsilon_{b} \\ \left(\epsilon_{\mathrm{th}} / \epsilon_{b}\right)^{\alpha} & \epsilon_{\mathrm{th}} \geq \epsilon_{b}\end{cases}
$$

For sufficiently high $\epsilon_{\mathrm{th}}$, it is possible for $\eta_{\mathrm{th}} \gg 1$, implying that $\ell_{\gamma \gamma} \gg \ell_{\text {IC }}$. When $\eta_{\text {th }}=1$, this is larger than $\ell_{\text {IC }}$ by roughly a factor of three, implying in this case that the gap scale height comparable to either. A more quantitative model of the gap lepton population may be obtained in one-dimension by assuming that the pairs are accelerated instantaneously to their asymptotic velocities. As shown in Appendix B.1, this implies a gap thickness of

$$
\Delta \approx \sqrt{2 \ell_{\mathrm{IC}} \ell_{\gamma \gamma}} \approx 1.7 \times 10^{10} R_{15}^{-1 / 2} \Omega_{F,-4}^{-1 / 2} B_{2}^{-1 / 2} u_{s, 0}^{-1 / 2} \eta_{\mathrm{th}}^{1 / 2} \mathrm{~cm}
$$

consistent with this. Generally, $\ell_{\gamma \gamma}$ is larger than $\ell_{\mathrm{IC}}$ and therefore the asymptotic Lorentz factor is gained on a length scale $\ell_{\text {IC }} \ll \Delta$ thereby justifying our assumption that the pairs are instantaneously accelerated.

Counter-propagating positrons or electrons constantly reinitiate the cascade, which continues until sufficient charges are produced to screen the electric fields. Thus we would anticipate that the gap can be evacuated only over a single scale height. The top of the gap is then roughly defined when the charge density is sufficient to generate the gap electric field gradients, and thus the lepton density in the lab frame can be expressed as (see Appendix B.1):

$$
n_{\mathrm{g}}=\frac{\nabla \cdot \mathbf{E}}{4 \pi e} \approx \frac{E}{4 \pi e \Delta}=3.3 R_{15}^{3 / 2} \Omega_{F,-4}^{3 / 2} B_{2}^{3 / 2} u_{s, 0}^{1 / 2} \eta_{\mathrm{th}}^{-1 / 2} \mathrm{~cm}^{-3}
$$

Note that this exceeds the Goldreich-Julian density by a multiplicity factor given by

$$
\eta=\frac{R}{\Delta}
$$

resulting from the smaller typical scale set by the gap thickness. $^{2}$ Here we have assumed that only a single species is present at the top of the gap, an assumption that is justified by the rapid acceleration of electrons and positrons in opposite directions. However, the nonlinear response of the magnetosphere will supply the charges of the opposite sign to screen the gap electric field and ensure that the microphysical current matches the global magnetospheric current (which is generically of order of Goldreich-Julian current, $\left.j_{\mathrm{GJ}}=e n_{\mathrm{GJ}} c\right)$.

We note that first-principles particle-in-cell (PIC) simulations are needed in order to simulate the cascade in detail and properly compute the flow of charges and connection between

\footnotetext{
2 This factor was not included in previous works (e.g., Levinson \& Rieger 2011; Mościbrodzka et al. 2011).
} 
the pair cascade and global MHD currents. Fortunately, our simplified, steady-state consideration of the gap structure appears to capture the most crucial aspects of the gap structure to within an order of magnitude. For instance, in PIC simulations of polar cascades in pulsar magnetosphere (Timokhin \& Arons 2013, hereafter, TA13), the simulated pair multiplicity $\eta \sim 100$ (for their polar gap thickness of $\Delta \sim 1.7 \times 10^{4} \mathrm{~cm}$ and a characteristic length scale $R=10^{6} \mathrm{~cm}$; see, e.g., Figure 22 in TA13) agrees with our estimate (19), which gives $\eta=R / \Delta \approx 60$. Furthermore, in agreement with the findings of TA13, our gap thickness is much larger than the skin depth,

$$
\frac{\Delta}{d_{e}}=5.6 \times 10^{5} \eta_{\mathrm{th}}^{1 / 4} R_{15}^{1 / 4} \Omega_{F,-4}^{1 / 4} B_{2}^{1 / 4} u_{s, 0}^{-1 / 4},
$$

with the skin depth given by

$$
d_{e}=\sqrt{\frac{m c^{2}}{4 \pi e^{2} n_{g}}} .
$$

Encouraged by this agreement, we will adopt the results of our steady state gap model and consider astrophysical consequences.

The relativistic outflow of particles at the top of the gap can carry a substantial total kinetic luminosity. Given the above estimates for the number density and typical Lorentz factor, the kinetic flux in the outflowing leptons is

$$
\begin{aligned}
F_{\text {lep }} & =\gamma_{\text {max }, \text { IC }} m_{e} c^{3} n_{\mathrm{g}} \\
& =3.4 \times 10^{13} R_{15}^{2} \Omega_{F,-4}^{2} B_{2}^{2} \eta_{\mathrm{th}}^{-1 / 2} \mathrm{erg} \mathrm{cm}^{-2} \mathrm{~s}^{-1} .
\end{aligned}
$$

Integrating this across the entire stagnation surface gives a total kinetic luminosity of

$$
\begin{aligned}
L_{\text {cascade }} & \approx \int_{0}^{R} 4 \pi R^{\prime} d R^{\prime} F_{\text {lep }} \\
& =1.1 \times 10^{44} R_{15}^{4} \Omega_{F,-4}^{2} B_{2}^{2} \eta_{\text {th }}^{-1 / 2} \mathrm{erg} \mathrm{s}^{-1} .
\end{aligned}
$$

Note that typically $\Omega_{F} \propto M^{-1}$ and $R \propto M$, where $M$ is the mass of the black hole. Hence, at fixed magnetic field strength the pair luminosity scales as $M^{2}$, implying that $L_{e}$ lep $/ L_{\text {Edd }} \propto M$. Our fiducial numbers correspond roughly to M87, and thus a black hole mass of order $10^{10} M_{\odot}$, for which the above luminosity is about $3 \times 10^{-4} L_{\mathrm{Edd}}$. This suppresses the cascade luminosity for less massive black holes, as we will see on an example of Sgr A* in $\$ 5$.

\subsection{Gap Stability}

The lepton structure in the gap is generally quite unstable, both due to the large-scale GRMHD processes that lead to its formation and the particle acceleration that fill it. In the case of its generic structure, the typical variability timescale is of order the light crossing time of the radial position of the gap, typically $\approx 10 r_{\mathrm{g}}$. The radial position of the gap varies by about $\lesssim 10 \%$ on this time scale, as can be seen in Figure 2 .

The particle acceleration processes will induce local variability on much shorter timescales, comparable to the light crossing time across the gap (see Appendix B.2). In our fiducial model, this is roughly $0.7 R_{15}^{-1 / 2} \Omega_{F,-4}^{-1 / 2} B_{2}^{-1 / 2} u_{s, 0}^{-1 / 2} \mathrm{~s}$. For M87, this is roughly six orders of magnitude smaller than $G M / c^{3}$ ! However, in practice, the gap has a transverse extent that greatly exceeds $\Delta$, and therefore usually many independently fluctuating regions are visible at once. Assuming that each independent
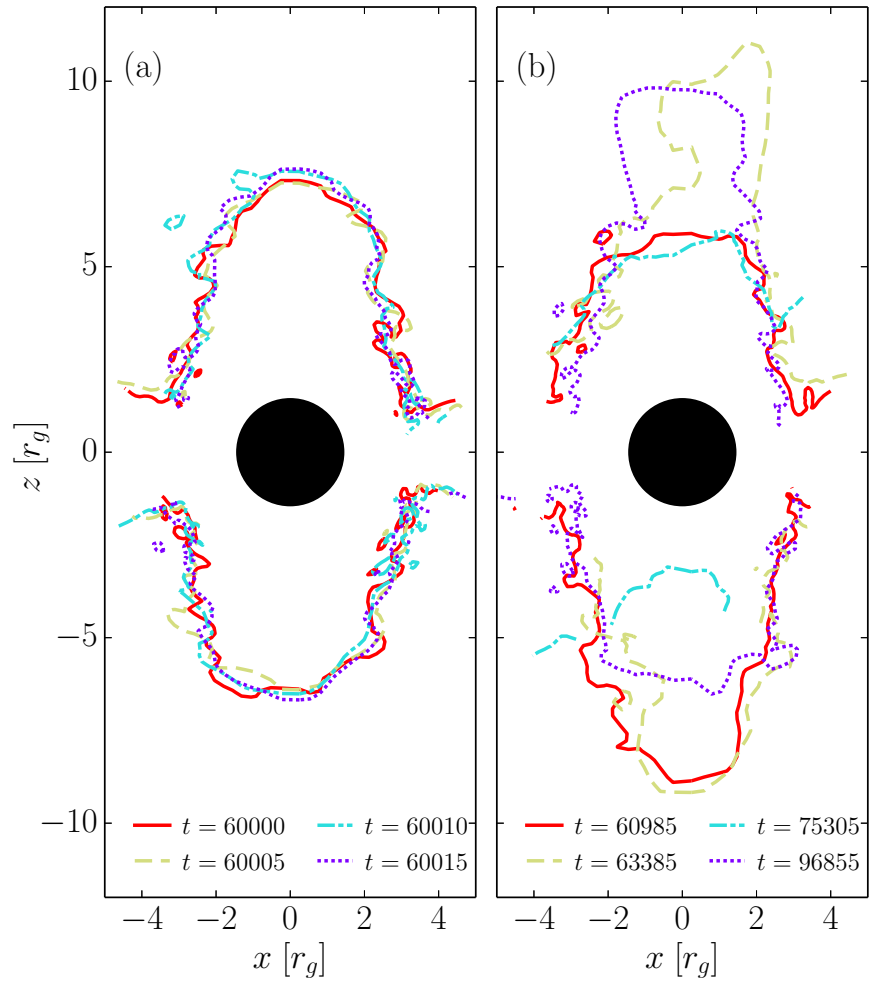

Figure 2. Meridional $(x-z)$ snapshots of the stagnation surface, at which the radial velocity vanishes $\left(u^{r}=0\right)$, shown at different times with different colors (see legend) for a simulation of magnetically-arrested disk around a spinning black hole with dimensionless spin $a=0.9$ (Tchekhovskoy et al., in preparation). For clarity, we only show the highly magnetized region, $p_{\mathrm{mag}} / \rho c^{2}>10$. The black hole event horizon is shown as the black filled circle. Panel (a) shows a series of snapshots of the stagnation surface taken at intervals comparable to the light crossing time, $\Delta t \approx 5 r_{\mathrm{g}} / c$. The stagnation surface is clearly variable on such a short timescale, with its radial position changing by $5-10 \%$. On average, the stagnation surface is at a distance $r \approx 5-10 r_{\mathrm{g}}$. Panel (b) shows how the shape of the stagnation surface evolves on longer time scales, $\Delta t \approx 10^{4} \mathrm{rg} / \mathrm{c}$. On such longer time scales the amplitude of variability can be as high as $50 \%$. For example, the shape of the surface can be substantially distorted by infalling gas that pushes the jet (e.g., see cyan dash-dotted line, which corresponds to $\left.t=75305 r_{\mathrm{g}} / c\right)$.

region has a local radius of $\Delta$, if the visible extent of the stagnation surface is $R_{V}$, the net degree of variability on the particle variation timescale is roughly

$$
\frac{\delta n_{\mathrm{g}}}{\left\langle n_{\mathrm{g}}\right\rangle} \approx \frac{\Delta}{R_{V}} .
$$

For low frequencies, $R_{V} \approx R$, and this reduction is enormous. However, for the very-high energy gamma rays produced directly by inverse Compton scattering, the intrinsic relativistic beaming will substantially reduce $R_{V}$. For emission associated with electrons with Lorentz factor $\gamma$, a patch of the surface that will be visible has a typical size of $R / \gamma$, implying that for $\gamma \gtrsim 10^{6}$ the observed particle density variations, and therefore the flux variations, will be of order unity on the particle acceleration timescale. In our fiducial model, where the soft seed photon energy density peaks around $1 \mathrm{meV}$, this corresponds to a gamma-ray energy above a few $\mathrm{GeV}$.

As the energy $\epsilon_{\gamma}$ of a gamma-ray increases, the number density of soft photons that lie above the pair creation threshold increases, and so does the optical depth $\tau_{\gamma \gamma}$ to pair production. The existence of the pair catastrophe implies that at sufficiently high $\epsilon_{\gamma}$ the optical depth to pair production is high, 
i.e., $\tau_{\gamma \gamma} \gg 1$. Since the energy threshold for annihilation of a gamma ray is $\propto \epsilon_{\gamma}^{-1}$, the number density of soft photons above the pair creation threshold (and therefore the optical depth to annihilation) scales as $\propto \epsilon_{\gamma}^{\alpha}$. The normalization of the total gamma-ray optical depth can be obtained in terms of $\ell_{\gamma \gamma}$, set by $\tau_{\gamma \gamma}=1$ for gamma rays with energy $\gamma_{\max , I C} m_{e} c^{2}$ :

$$
\tau_{\gamma \gamma}\left(\epsilon_{\gamma}\right) \approx \frac{r}{\ell_{\gamma \gamma}} \frac{n_{s}\left(\epsilon_{s}>4 m_{e}^{2} c^{4} / \epsilon_{\gamma}\right)}{n_{s}\left(\epsilon_{s}>4 m_{e} c^{2} / \gamma_{\max , \text { IC }}\right)} \approx \frac{r}{\ell_{\gamma \gamma}}\left(\frac{\epsilon / m_{e} c^{2}}{\gamma_{\max , \text { IC }}}\right)^{\alpha} .
$$

Hence, for gamma-rays with energies

$$
\epsilon_{\gamma} \lesssim \epsilon_{\gamma, \min } \equiv \gamma_{\max , \mathrm{IC}} m_{e} c^{2}\left(\frac{\ell_{\gamma \gamma}}{r}\right)^{1 / \alpha}
$$

the seed photon distribution will be optically thin to the resulting inverse Compton gamma rays, allowing the latter to escape. For values typical of M87, this corresponds to energies of or$\operatorname{der} \epsilon_{\gamma} \approx 630 \mathrm{GeV}$, above which significant attenuation can be expected.

\section{PARTICLE CREATION AND ACCELERATION BEYOND THE STAGNATION SURFACE}

The leptons streaming out of the gap opened near the stagnation surface will still have near-maximal Lorentz factors, $\gamma_{\max \text {.IC. }}$ As a result, they will continue to pair produce beyond the gap each time they traverse a distance of roughly $\ell_{\text {IC }}$. However, unlike the results of the previous section, since the leptons are outside of the gap, they are no longer rapidly re-accelerated, and thus the pair catastrophe is no longer being driven. The result is a redistribution of the energy of gap-injected leptons among a set of daughter leptons produced via the resulting final cascade.

\subsection{Qualitative Asymptotic Estimates}

We have already noted that for gamma rays with energies below a lower limit determined by the seed photon SED the ambient soft photon population will be transparent, halting the pair production cascade. Explicitly, for a power law seed photon distribution above a low-energy cutoff $\epsilon_{b}$ (as described above Equation 16) the fraction of gamma rays with energies above $\epsilon_{\gamma, \text { min }}$ following a single scattering is

$$
f_{>\epsilon_{\gamma, \min }}(\gamma) \approx \begin{cases}\left(\epsilon_{\gamma, \min } / 2 \gamma^{2} \epsilon_{b}\right)^{-\alpha} & \gamma \leq \sqrt{\epsilon_{\gamma, \min } / 2 \epsilon_{b}} \\ 1 & \text { otherwise, }\end{cases}
$$

where we have assumed that $\epsilon_{b}$ is sufficiently small. This is necessarily a strong function of the initial lepton Lorentz factor, dropping rapidly for Lorentz factors below $\sqrt{\epsilon_{\gamma, \min } / 2 \epsilon_{b}}$, and thus $\epsilon_{\gamma, \text { min }}$ implies a limit upon $\gamma$.

However, since the energies of the upscattered gamma rays are typically small in comparison to the lepton energy near this limit, i.e., $2 \gamma^{2} \epsilon_{b} \ll \gamma m c^{2}$, in practice the lepton will undergo many scatterings before cooling appreciably. That is, there will be roughly

$$
N_{\mathrm{scat}} \approx \frac{\gamma m c^{2}}{2 \gamma^{2} \epsilon_{b}}=\frac{m c^{2}}{2 \gamma \epsilon_{b}},
$$

opportunities to produce a gamma ray with energy above $\epsilon_{\gamma \text {,min }}$. This increases with decreasing $\gamma$ and $\epsilon_{b}$, ameliorating the decrease in $f_{>\epsilon_{\gamma, \min }}$ due to the latter. Thus the number of pair producing gamma rays is expected to be approximately $N_{\text {scat }} f_{>\epsilon_{\gamma, \text { min }}}$. Setting this number to unity provides the desired limit upon the lepton Lorentz factor, below which electrons cease to typically have sufficient energy to continue the pair cascade:

$$
\gamma_{\gamma \gamma} \approx\left(\frac{2^{1-\alpha} \epsilon_{b}^{1-\alpha} \epsilon_{\gamma, \min }^{\alpha}}{m c^{2}}\right)^{1 /(2 \alpha-1)}
$$

For our fiducial model, $\gamma_{\gamma \gamma} \approx 7.7 \times 10^{4}$. This limit on the redistribution of energy implies an associated limit on increase in the particle density. An even redistribution, maximizing the number of leptons produced, results in

$$
\frac{n_{\infty}}{n_{\mathrm{g}}} \approx \frac{\gamma_{\max , \mathrm{IC}}}{\gamma_{\gamma \gamma}}
$$

and is roughly 6000 daughter leptons for each lepton produced by the gap for our fiducial model.

The absence of the generation of energetic gamma rays does not imply the absence of additional inverse-Compton cooling. Hence, a second, typically more stringent limit arises from inverse-Compton cooling directly. For the cooling length to become comparable to the scale height of the seed photon distribution requires

$$
\frac{3 m_{e} c^{2}}{4 \sigma_{T} u_{s} \gamma_{\infty}} \gtrsim r \Rightarrow \gamma_{\infty} \lesssim \gamma_{\max , \mathrm{IC}} \frac{\ell_{\mathrm{IC}}}{r} \approx 10^{2} u_{s, 0}^{-1} r_{16}^{-1}
$$

We note that in our fiducial model, the cooling of the leptons from $\gamma_{\gamma \gamma}$ to $\gamma_{\infty}$ is unable to produce gamma rays energetic enough to pair produce. Therefore, the resulting radiation can escape and can be a substantial source of emission.

Since $\gamma_{\infty} n_{\infty} \ll \gamma_{\max , I C} n_{\mathrm{g}}$ most of the energy imparted to the leptons by the accelerating electric field across the gap has been radiated via the Comptonization of the soft seed photons. Typical energies range from the optical to the GeV, with an SED that depends upon that of the underlying soft photon population. However, this emission will be highly beamed along the magnetic field lines that govern the particle motions.

\subsection{Numerical Asymptotic Estimates}

In practice the estimates obtained in the previous section depend critically upon assumptions regarding the final electron spectrum, that is itself dependent upon the soft photon SED. For this reason we also computed the asymptotic number and energy distribution of electrons produced due to inverse-Compton scattering off of a given seed photon SED from a nearly mono-energetic injection at high Lorentz factor. Throughout this section, for compactness we will measure energies in units of the electron rest mass energy.

We make two simplifying assumptions, both of which are almost certainly well justified. The first is that we may ignore any subsequent pair annihilation due the comparatively high lepton energies and low lepton densities. The second is that the number density of soft seed photons vastly exceeds the number densities of up-scattered gamma rays, a consequence of the short mean free path to pair production and the low lepton densities. These remove the non-linear terms in the coupled Boltzmann equations for the leptons and the gamma-rays. Thus, it is sufficient to consider the evolution of a single lepton through multiple Compton generations. That is, we compute the energy probability distribution of a single lepton as a function of the number of scatterings instead of time. The asymptotic form of this probability distribution then describes the asymptotic spectrum of a full population of leptons.

Explicit inputs are the seed photon spectrum and electron injection energy, which we leave as a parameter, $\epsilon_{0}$. For the 
former we assume a power law model between some minimum $\left(\epsilon_{m}\right)$ and maximum $\left(\epsilon_{M}\right)$ energies, consistent with the observed radio and infrared emission from the sources of interest, i.e.,

$$
f_{s}(\epsilon) \equiv \frac{\mathrm{d} N_{s}}{\mathrm{~d} \epsilon} \propto \begin{cases}\epsilon^{-\alpha-1} & \epsilon_{m}<\epsilon<\epsilon_{M} \\ 0 & \text { otherwise. }\end{cases}
$$

The values of $\epsilon_{m}$ and $\epsilon_{M}$ are determined by the particular source under consideration, though typically when a large enough dynamic range is chosen the resulting evolution becomes insensitive to their specific choice. ${ }^{3}$ The linearity in the gamma-ray distribution is synonymous with assuming that $f_{s}$ remains fixed throughout the post-gap pair cascade.

With the above we may now compute the update in the lepton energy distribution with each scattering. We do this in two steps, first computing the implied up-scattered gamma-ray distribution associated with a mono-energetic electron distribution, and then from that inferring the changes to the electron distribution arising from inverse-Compton losses and pair production. To compute the former, we begin with the relationship between the original seed photon energy $\epsilon_{s}$, up-scattered gamma-ray energy $\epsilon_{\gamma}$, and initial electron energy $\epsilon_{e, i}$ :

$$
\epsilon_{s}\left(\epsilon_{\gamma}, \epsilon_{e, i}\right)= \begin{cases}\frac{\epsilon_{\gamma}}{2 \epsilon_{e, i}\left(\epsilon_{e, i}-\epsilon_{\gamma}\right)} & \epsilon_{\gamma}<\epsilon_{e, i} \\ 0 & \text { otherwise }\end{cases}
$$

where we assumed $\epsilon_{S} \ll \epsilon_{e, i}$ and approximated energy of the electron post-collision as $\epsilon_{e, f}=\epsilon_{e, i}-\epsilon_{\gamma}$. Equation (31) may be obtained from the standard Compton scattering formula in the high-energy electron and oblique seed photon limits. As a result, for a monoenergetic electron distribution, the up-scattered gamma-ray distribution is

$$
g\left(\epsilon_{\gamma}, \epsilon_{e, i}\right)=\frac{d \epsilon_{s}}{d \epsilon_{\gamma}} f_{s}\left[\epsilon_{s}\left(\epsilon_{\gamma}, \epsilon_{e, i}\right)\right]=\frac{f_{s}\left[\epsilon_{s}\left(\epsilon_{\gamma}, \epsilon_{e, i}\right)\right]}{2\left(\epsilon_{e, i}-\epsilon_{\gamma}\right)^{2}} .
$$

As mentioned above, since $\epsilon_{s} \ll \epsilon_{e, i}, \epsilon_{\gamma}$, we approximate the electron energy following scattering by $\epsilon_{e, f} \approx \epsilon_{e, i}-\epsilon_{\gamma}$. Therefore, if $f_{j}\left(\epsilon_{e}\right)$ is the electron distribution after the $j$ th scatter, the electron distribution is rearranged by inverse-Compton losses to

$$
f_{\mathrm{IC}, j+1}^{\mathrm{lep}}\left(\epsilon_{e}\right)=\int_{0}^{\infty} d \epsilon_{e, i} g\left(\epsilon_{e, i}-\epsilon_{e}, \epsilon_{e, i}\right) f_{j}^{\mathrm{lep}}\left(\epsilon_{e, i}\right) .
$$

In practice the integral need only be computed up to the initial maximum energy of the electron distribution.

Similarly, since $\epsilon_{\gamma} \gg 2 \gg \epsilon_{s}$, i.e., much greater than the rest mass of the produced pair, which is itself much greater than that of the seed photons, we may approximate the energy of each lepton in the resulting pair by $\epsilon_{e}=\epsilon_{\gamma} / 2$. Hence, the distribution of new pairs is

$$
f_{\gamma \gamma, j+1}^{\mathrm{lep}}\left(\epsilon_{e}\right)=4 \int_{0}^{\infty} d \epsilon_{e, i} g\left(2 \epsilon_{e}, \epsilon_{e, i}\right) f_{j}^{\mathrm{lep}}\left(\epsilon_{e, i}\right) \Theta\left(2 \epsilon_{e}-\epsilon_{\gamma, \min }\right),
$$

where $\Theta(x)$ is the Heaviside function and ensures that the integral is only over regions in which the gamma-ray can pair produce on the soft background. Note that we assume that all gamma-rays will pair produce as long as their energy exceeds

\footnotetext{
${ }^{3}$ The reason is that when $\epsilon_{m} \ll 4 m_{e}^{2} c^{4} / \epsilon_{0}$ the up-scattered photons will be unable to pair produce (and the energy loss becomes comparatively insignificant) and when $\epsilon_{M} \gg 4 m_{e}^{2} c^{4} / \epsilon_{0}$ the number density of photons with energies near $\epsilon_{M}$ will be small in comparison to those responsible for the bulk of the particle creation (and thus may be neglected).
}

the threshold $\epsilon_{\gamma, \min }$, above which the seed photon distribution is optically-thin to pair production.

In principle, we should then set $\epsilon_{M}$ by the pair production optical depth; from Equation (24) this gives a typical

$$
\epsilon_{M} \approx 4 m_{e}^{2} c^{4} /(630 \mathrm{GeV})
$$

which for our fiducial parameters gives $\epsilon_{M} \approx 1.6 \mathrm{eV}$. In practice, we find the simulation results are insensitive the particular value we choose for $\epsilon_{\mathrm{M}}$. In all of our simulations, unless specified otherwise, we choose $\epsilon_{M}=0.8 \mathrm{eV}$ (as we discuss below, results for $\epsilon_{M}=1.6 \mathrm{eV}$ are essentially identical; see Figure 6). In a similar fashion to Equation (34), we can write down the distribution function of photons that are below the pair production threshold, $\epsilon_{\gamma}<\epsilon_{\gamma, \min }$ : these photons escape the system without undergoing scattering and thus form the high-energy emission spectrum,

$$
f_{\gamma \gamma, j+1}^{\mathrm{ph}}\left(\epsilon_{\gamma}\right)=4 \int_{0}^{\infty} d \epsilon_{e, i} g\left(\epsilon_{\gamma}, \epsilon_{e, i}\right) f_{j}^{\mathrm{lep}}\left(\epsilon_{e, i}\right) \Theta\left(\epsilon_{\gamma, \min }-\epsilon_{\gamma}\right) .
$$

Combining the above, we obtain the energy distribution of the leptons and photons in the $(j+1)$ th generation via

$$
\begin{aligned}
& f_{j+1}^{\mathrm{lep}}\left(\epsilon_{e}\right)=f_{\mathrm{IC}, j+1}^{\mathrm{lep}}\left(\epsilon_{e}\right)+f_{\gamma \gamma, j+1}^{\mathrm{lep}}\left(\epsilon_{e}\right), \\
& f_{j+1}^{\mathrm{ph}}\left(\epsilon_{\gamma}\right)=f_{j}^{\mathrm{ph}}\left(\epsilon_{\gamma}\right)+f_{\gamma \gamma, j+1}^{\mathrm{ph}}\left(\epsilon_{\gamma}\right) .
\end{aligned}
$$

Beginning with some initial injection distribution, we can compute the asymptotic distribution, in which generations correspond in a loose sense to time or height from the injection point.

For this, we integrate Equations (37)-(38) numerically. We start the integration at the top of the gap, and inject a single electron at an energy

$$
E_{0}=\gamma_{\max , \mathrm{IC}} m_{e} c^{2}
$$

which is the energy gained by an electron between its consequent encounters of seed photons in the gap and is therefore the characteristic energy with which the electrons emerge from the gap. We discretize Equations (37)-(38) on a logarithmic grid in energy, which extends from $E_{\min }=10^{-6} m_{e} c^{2}$ to $E_{\max }=2 E_{0}$. For numerical reasons, we smooth out the initial energy distribution by $1.5 \%$ so it is properly represented on our numerical grid, by choosing the following initial injection distribution,

$$
f_{0}^{\text {lep }}\left(\epsilon_{e}\right) \equiv \frac{\mathrm{d} N_{e}}{\mathrm{~d} \epsilon_{e}}=\frac{1}{\sqrt{2 \pi \sigma_{E}^{2}}} \exp \left[-\frac{\left(\epsilon_{e}-E_{0}\right)^{2}}{2 \sigma_{E}^{2}}\right],
$$

with $\sigma_{E}=0.015 E_{0}$.

In order to ensure robust evolution of pair cascade numerically, we found it to be important to ensure lepton number conservation under Inverse Compton cooling (Equation (33)). To do this, we compute the discretization of the integral in Equation (33) on two energy grids that contain the same number of grid cells but are shifted relative to each other by half a cell. If the changes in the number of leptons due to these two discretizations are different by more than $50 \%$ relative to each other, then we use their weighted sum that preserves the total number of leptons exactly. Otherwise, we use the discretization that results in the smallest change in lepton number. We implemented the numerical code in a Cython-based Python library. In order to speed up the integrations, we parallelized the code using OpenMP. We describe the numerical results of cascade evolution in Section 4.3. 


\section{APPLICATION TO M87}

Thus far we have remained agnostic regarding the particular sources for which particle acceleration at the stagnation surface is relevant. Here we apply this model to the generation of the nonthermal particles in the radio jet of M87. We begin with a short overview of the relevant source parameters and their corresponding gap parameters.

\subsection{M87 Source Parameters}

Observations of stellar dynamics within M87's sphere of influence produce an inferred mass of $6.6 \times 10^{9} M_{\odot}$, assuming a distance of $17.9 \mathrm{Mpc}$ (Gebhardt et al. 2011). ${ }^{4}$ The energy output of M87 peaks at $1 \mathrm{~mm}(300 \mathrm{GHz}$ or $1 \mathrm{meV})$, at which point the flux at Earth is roughly $1 \mathrm{Jy}$. The typical isotropicequivalent total luminosity is $L \approx 10^{42} \mathrm{erg} \mathrm{s}^{-1} \approx 10^{-6} L_{\mathrm{Edd}}$, where $L_{\text {Edd }}$ is the Eddington luminosity. This makes M87 quite underluminous and dominated by emission at wavelengths about the peak frequency, as we discuss below. The typical scale near the black hole is the gravitational radius of the black hole, $r_{\mathrm{g}} \simeq 10^{15} \mathrm{~cm}$. Here we discuss the empirical implications of various additional observations for the physical properties near the stagnation surface.

\subsubsection{Jet Velocity and Orientation}

Apparent motions near the black hole of roughly $2 c$ have been observed (e.g., Walker et al. 2008). These imply a minimum Lorentz factor of $\Gamma \approx 2.3$ by distances of 0.3 mas, corresponding to linear distances of $8 \times 10^{16} \mathrm{~cm} \approx 80 r_{\mathrm{g}}$, and implying an inclination of $25^{\circ}$. On scales of $1^{\prime \prime}$, more than three orders of magnitude farther out, apparent motions of $6 c$ have been observed, implying $\Gamma>6$ at an inclination of $\approx 10^{\circ}$. Here we will employ the radio implied Lorentz factor and associated orientation, $\Gamma=2.3$ and $\Theta=25^{\circ}$, where specific values are required.

\subsubsection{Magnetic Field Strength Estimates}

The observational implications for the magnetic field in M87 depend upon the structure and dynamics of the material in the vicinity of the black hole. Here we present two estimates, assuming in the first that the observed mm-wavelength emission is due to a radiatively inefficient accretion flow (RIAF, Narayan \& Yi 1994, 1995), and in the second that the jet is Poynting dominated and responsible for the power observed in the radio lobes. Both reach similar conclusions regarding the field strength.

As suggested by their name, RIAFs are characterized by their extraordinarily low radiative efficiency, i.e., $L=\eta_{d} \dot{M} c^{2}$ with $\eta_{d} \approx 10^{-3}-10^{-4}$. Since most of the gravitational binding energy is released, and therefore most of the luminosity is produced, near the black hole, this implies an accretion flow density of

$$
\rho \approx \frac{\dot{M}}{4 \pi r_{\mathrm{g}}^{2} \beta c}=\frac{L}{4 \pi \eta_{d} r_{\mathrm{g}}^{2} \beta c^{3}}
$$

where we have assumed a disk height of $r$ and an inflow velocity near the horizon of $\beta c$. The jet magnetic field will accumulate until it balances the ram pressure of the accretion flow, and

\footnotetext{
${ }^{4}$ While quantitative differences appear if we were to adopt the lower mass of $3.5 \times 10^{9} M_{\odot}$ implied by gas dynamical modeling (Walsh et al. 2013), the qualitative consequences remain unchanged.
}

thus we arrive at the first magnetic field strength estimate of

$$
B \approx \sqrt{8 \pi \rho \beta^{2} c^{2}} \approx\left(\frac{2 L \beta}{\eta_{d} r_{\mathrm{g}}{ }^{2} c}\right)^{1 / 2} \approx 3 \times 10^{2} \eta_{d,-3}^{-1 / 2} L_{42}^{1 / 2} \beta^{1 / 2} \mathrm{G} .
$$

It is not clear that the mm-wavelength emission is associated with an accretion flow. At $7 \mathrm{~mm}$ the jet dominates the source morphology (Walker et al. 2008). Thus, we present a second estimate based upon the assumption that the jet is Poynting dominated near the black hole and relating the mechanical power at small and large radii. The Poynting luminosity associated with black-hole driven jets is (Tchekhovskoy et al. 2010):

$$
L_{\mathrm{EM}}=\frac{k}{4 \pi c} \Omega_{\mathrm{H}}^{2} \Phi^{2}
$$

where $k \approx 0.045$ for parabolic jet geometry, $\Phi$ is the poloidal magnetic flux threading the black hole, and $\Omega_{H}$ is the angular velocity of the horizon of the black hole, which encodes the dependence upon the black hole spin. ${ }^{5}$

A variety of estimates of the total jet power in M87 have been obtained across a wide range of scales. Radio lobe measurements probe emission extending over $30 \mathrm{kpc}$, and have resulted in estimates of $L_{\text {jet }} \approx 10^{44} \mathrm{erg} \mathrm{s}^{-1}$ (de Gasperin et al. 2012). This is similar to estimates arising from the modeling of the complex of knot features on kpc-scales (Owen et al. 2000), and on $60 \mathrm{pc}$ scales from interpreting the HST-1 complex as a recollimation shock (Stawarz et al. 2006). Thus, we adopt this estimate as a conservative estimate of the total jet power. For a characteristic black hole spin, $a=0.9$, equating these then gives a poloidal magnetic field strength estimate at the event horizon:

$$
B_{P}=\frac{\Phi}{A} \approx 2.5 \times 10^{2} \mathrm{G}
$$

where $A=2 \pi\left(a^{2} r_{g}^{2}+3 r_{\mathrm{H}}^{2}\right) / 3$ is the area of the one of the two hemispheres of the event horizon, where $r_{\mathrm{H}}=r_{\mathrm{g}}\left(1+\sqrt{1-a^{2}}\right)$ is the radius of the black hole event horizon.

Despite the significantly different physics being invoked, the two estimates are quite similar. While this is not accidental (thick disks are believed to be a necessary component of jet formation), it does critically depend upon the low radiative efficiency generally implicated in vastly sub-Eddington sources (such as M87).

At the distances of the stagnation surface, which lies inside the light cylinder, the magnetic field is poloidally-dominated. If we now assume that the jet is roughly parabolic, i.e., $R_{j} / r_{\mathrm{g}} \approx$ $\left(r / r_{\mathrm{g}}\right)^{1 / 2}$, within the gap region the above estimates imply

$$
B \approx \frac{\Phi}{\pi R_{j}^{2}} \approx 35 \mathrm{G}
$$

for a typical stagnation surface distance of $r \sim 10 r_{\mathrm{g}}$.

\subsubsection{Synchrotron Cooling Times} is

In the jet frame, the observed synchrotron cooling timescale

$$
t_{\mathrm{sync}}^{\prime}=\frac{3 m c^{2}}{4 \sigma_{T} u_{B}^{\prime} c \gamma^{\prime}}=\frac{6 \pi m c^{2}}{\sigma_{T} B^{\prime 2} c} \sqrt{\frac{\nu_{B}^{\prime}}{\nu^{\prime}}}=\frac{3}{\sigma_{T}}\left(\frac{2 \pi e m c}{B^{\prime 3} \nu^{\prime}}\right)^{1 / 2},
$$

5 Note that the low-spin approximation given by Equation (43) remains accurate for spins $a \lesssim 0.95$, beyond which it over-estimates the true power by up to $30 \%$. For a higher order expansion that remains accurate for all spins, see Tchekhovskoy et al. (2010). See also Tchekhovskoy et al. (2012) for the comparison of various approximation to jet power. 
where $\nu^{\prime}$ and $\nu_{B}^{\prime} \equiv e B^{\prime} / 2 \pi m_{e} c$ are the observation and cyclotron frequencies in the jet frame, and $\gamma^{\prime}$ is the Lorentz factor of nonthermal particles, again measured in the jet frame. To relate this to emission in the lab frame, note that $\nu^{\prime}=$ $\nu \Gamma(1-\beta \cos \Theta)$ and since the magnetic field is likely to be dominantly toroidal, $B^{\prime} \approx B / \Gamma$. Hence, as measured in the lab frame,

$$
\begin{aligned}
t_{\mathrm{sync}}=t_{\mathrm{sync}}^{\prime} \Gamma & \approx \frac{3}{\sigma_{T}}\left[\frac{2 \pi e m c \Gamma^{4}}{B^{3} \nu(1-\beta \cos \Theta)}\right]^{1 / 2} \\
& \approx 1.8 \times 10^{5} B_{1.5}^{-3 / 2} \nu_{11.5}^{-1 / 2} \mathrm{~s},
\end{aligned}
$$

where $B=30 B_{1.5} \mathrm{G}$ and $\nu=300 \nu_{11.5} \mathrm{GHz}$ and the remainder of the quantities are given by our fiducial M87 values.

Evident in Equation (47) is the strong dependence of $t_{\text {sync }}$ on $B$ and $\Gamma$, and therefore position. As the jet propagates $B$ decreases and $\Gamma$ increases in a fashion that depends on the specific jet structure, resulting in a corresponding rapid increase in $t_{\text {sync }}$. Generally, magnetic flux conservation implies that the poloidal and toroidal components of the magnetic field within the jet scale $\propto R^{-2}$ and $\propto R^{-1}$, respectively (it is for this reason that despite having similar strengths near the black hole, jet magnetic fields quickly become strongly toroidally dominated). For a parabolic magnetic field, $R \propto z^{1 / 2}$, which we assumed when obtaining Equation (47). Similarly, $\Gamma$ is expected to rise $\propto R$. However, in practice the Lorentz factor of the jet in M87 appears to asymptote to $\approx 5$ at large radii, limiting its impact. Regardless, near the stagnation surface these imply that $t_{\text {sync }}$ will initially grow $\propto z^{7 / 4}$, eventually slowing to $\propto z^{3 / 4}$.

The implication for the image structure is obtained by comparing $t_{\text {sync }}$ to the outflow timescale,

$$
t_{\text {outflow }}=\frac{z}{\beta c} \approx 3.3 \times 10^{5}\left(\frac{r}{10 r_{\mathrm{g}}}\right) \mathrm{s},
$$

which grows linearly with jet height. When $t_{\text {sync }}>t_{\text {app }}$ the relativistic leptons cannot efficiently cool, and therefore the nonthermal particle density is effectively conserved. At moderate distances from jet base $\left(z \gtrsim 15 r_{\mathrm{g}}\right)$ the above estimates suggest that this is the case. At much larger distances where the Lorentz factor saturates $\left(z \gtrsim 10^{3} r_{\mathrm{g}}\right)$ the synchrotron cooling becomes efficient.

Near the stagnation surface $t_{\text {sync }}$ is comparable to $t_{\text {outflow }}$. However, this is complicated by the large initial bulk Lorentz factors resulting from the acceleration across the gap. As a result, a substantial fraction of leptons still resides in the ordered non-thermal relativistic component that emerged from the polar cascade propagating along magnetic field lines and therefore geometrically immune to synchrotron cooling. The study of how these nonthermal electrons isotropize and cool is beyond the scope of this paper. Thus, for simplicity we will ignore synchrotron cooling in the estimation of the energetic lepton population of the jet.

\subsubsection{Seed Photon Density Estimates}

The source of the soft seed photons in M87 is not immediately clear due to potential contamination from the jet itself. Nonetheless, it is now certain that the millimeter-wavelength emission is associated with compact features with scales comparable to that of the horizon (Doeleman et al. 2012). This provides circumstantial evidence that the observed millimeter, infrared, and optical emission arise very near the black hole itself. Thus, even if the emission is from within the jet itself, at the jet heights of relevance the bulk Lorentz factors are expected to be small (of order unity), and hence not strongly beamed. As a consequence, we estimate of the seed photon density from the observed emission directly.

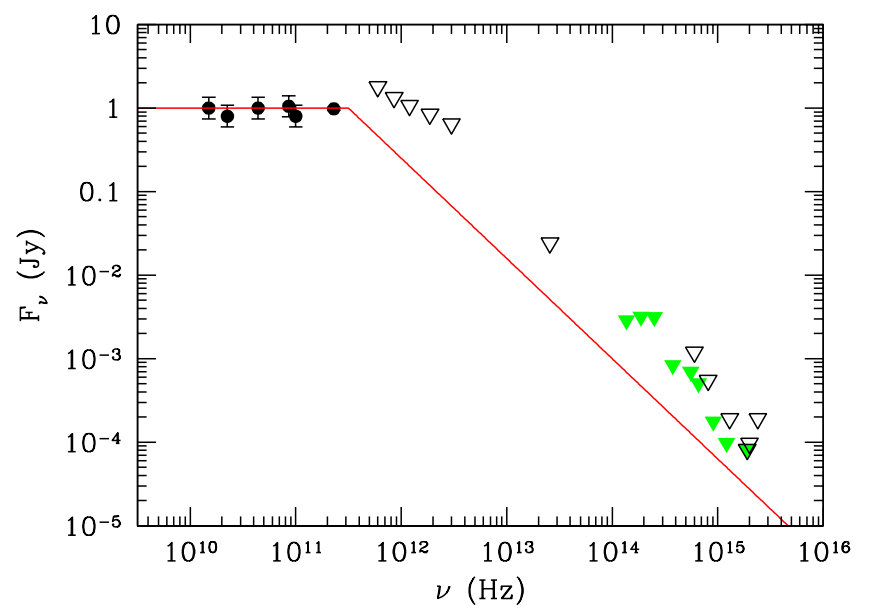

Figure 3. Spectral energy distribution for M87 from the radio to the optical, constructed from the literature (Spencer \& Junor 1986; Baath et al. 1992; Junor \& Biretta 1995; Sparks et al. 1996; Lonsdale et al. 1998; Biretta et al. 2002; Ly et al. 2004; Kovalev et al. 2005; Lister \& Homan 2005; Petrov et al. 2007; Perlman et al. 2007; Lee et al. 2008; Baes et al. 2010; Doeleman et al. 2012, additional details will be published elsewhere). Green filled triangles show a subset of the infrared-optical flux limits collected in Broderick et al. (2015). Filled circles and open triangles correspond to flux measurements in which the source is resolved and unresolved, respectively; we take the filled circles as directly indicative of the near-horizon seed photon distribution and the open triangles as upper limits. Errorbars indicate variability, not intrinsic measurement uncertainty. For comparison, a simple broken power-law with high-frequency spectral index $\alpha=1.2$, as employed in the text, is shown for reference.

The SED of M87, shown in Figure 3, is reasonably well fit by a broken power law, i.e., $F_{\nu} \propto \nu^{-\alpha}$ where $\alpha$ evolves rapidly as the source becomes optically thin (see Fig. 3):

$$
\alpha \approx \begin{cases}0 & \nu<300 \mathrm{GHz} \\ 1.2 & \nu \geq 300 \mathrm{GHz}\end{cases}
$$

The flux density at the break is roughly $1 \mathrm{Jy}$. If all of the emission above $300 \mathrm{GHz}$ arises in a radiatively inefficient accretion disk, then the soft photon energy density is roughly

$$
u_{s} \approx 0.01 \mathrm{erg} \mathrm{cm}^{-3}
$$

where we have now fixed the location of the stagnation surface to $10 r_{\mathrm{g}}$. This is well above the densities necessary for inverse Compton losses to dominate the limits upon the acceleration of the leptons within the gap.

\subsubsection{Implied Nonthermal Particle Properties}

If the sub-mm luminosity arises due to optically thin emission of the jet launching region, as suggested by the recent mm-VLBI observations (Doeleman et al. 2012), it implies a rather soft nonthermal particle density, with $d n / d \gamma \approx \gamma^{-3.4}$. The source size is roughly $40 \mu$ as, implying that the associated 
millimeter flux is related to the particle density by

$$
\begin{aligned}
F_{\mathrm{mm}} & \approx \frac{\sqrt{27} e^{2} \nu_{B}}{8 \pi^{2} c}\left(\frac{3 \nu_{B}}{\nu}\right)^{\alpha} \frac{2 \alpha \gamma_{\min }^{2 \alpha}}{\alpha+1} \Gamma\left(\frac{\alpha}{2}+\frac{11}{6}\right) \Gamma\left(\frac{\alpha}{2}+\frac{1}{6}\right) \Omega r n \\
& =4.9 \times 10^{-2}\left(\frac{\theta}{40 \mu \mathrm{as}}\right)^{2}\left(\frac{r}{10 r_{\mathrm{g}}}\right) n B_{1.5}^{1+\alpha} \mathrm{Jy},
\end{aligned}
$$

where $\nu_{B} \equiv e B / 2 \pi m_{e} c$ is the cyclotron frequency, $\Gamma(x)$ is the standard gamma-function, $\nu=300 \mathrm{GHz}$, and we have assumed a typical lower cutoff on the accelerated particle distribution of $\gamma_{\text {min }} \approx 10^{2}$. Comparing this to an observed flux of roughly $1 \mathrm{Jy}$ and assuming the magnetic field estimate in Equation (45) gives an approximate nonthermal particle density estimate of

$$
n \approx 15 \mathrm{~cm}^{-3}
$$

consistent with efforts to quantitatively model the emission region of M87 on horizon scales (Broderick et al., in preparation).

\subsection{Particle Acceleration at the Stagnation Surface}

We can now estimate the properties of the gap, and the associated accelerated particles, from M87's parameters given above. This gives our fiducial model for M87. The asymptotic lepton Lorentz factor is determined by inverse Compton losses and is roughly

$$
\gamma_{\max } \equiv \gamma_{\max , \mathrm{IC}} \approx 1.6 \times 10^{9},
$$

and this sets our fiducial value of electron energy, $E_{0}$. Whereas its numerical value is known only to a factor of few, we choose not to round this value in order to get a self-consistent answer. This corresponds to a seed photon pair-production threshold of

$$
\epsilon_{\mathrm{th}} \approx 1.2 \mathrm{meV} \quad \Rightarrow \quad \lambda_{\text {th }} \approx 1.1 \mathrm{~mm} .
$$

Therefore, photons at and just below the spectral break in M87's SED will contribute to pair production within the gap.

The length scale over which the asymptotic lepton Lorentz factors are obtained is (see Equation 6)

$$
\ell_{\mathrm{IC}} \approx 5.5 \times 10^{10} \mathrm{~cm}=5.6 \times 10^{-5} r_{\mathrm{g}}
$$

and the typical mean free path of the up-scattered photons to pair production is (see Equation 15)

$$
\ell_{\gamma \gamma} \approx 1.7 \times 10^{12} \mathrm{~cm} \approx 1.7 \times 10^{-3} r_{\mathrm{g}} .
$$

Hence, as anticipated, both the acceleration and subsequent pair production within the gap occurs on scales much smaller than the typical gap thickness that might be expected on global considerations (i.e., $r_{\mathrm{g}}$ ). The resulting gap thickness is then roughly (see Equation 17)

$$
\Delta \approx 4.3 \times 10^{11} \mathrm{~cm}
$$

implying a corresponding density at the top of the gap of (see Equation 18)

$$
n_{\mathrm{g}} \approx 2.2 \times 10^{-2} \mathrm{~cm}^{-3} .
$$

We note that since $\Delta$ by about an order of magnitude exceeds $\ell_{\text {IC }}$, over which the pairs attain their terminal Lorentz factor, our earlier assumption that the pairs instantaneously attain their terminal Lorentz factor is justified.

\subsection{Post-Gap Cascade}

Given the threshold seed photon energy of $1.2 \mathrm{meV}$, the minimum gamma-ray energy for which the seed photons bath is optically-thick to pair production is $\epsilon_{\gamma \text {,min }}=640 \mathrm{GeV}$, hence the asymptotic Lorentz factor for M87 is (see Equation 27)

$$
\gamma_{\gamma \gamma}=2.6 \times 10^{6}
$$

implying enhancement in the number density due to the postgap cascade of roughly $n_{\infty} / n_{g}=670$ (see Equation 28).

To verify this analytical estimate, we carried out numerical integration of the pair cascade equations (37)-(38), as described in Section 3.2. We choose the following parameters to describe the seed photon spectrum in our fiducial model of M87: $\alpha=1.2, \epsilon_{m}=1.2 \mathrm{meV}, \epsilon_{M}=0.8 \mathrm{eV}$. We start with an initial distribution that corresponds to an electron emerging from the top of the gap at an energy $E_{0}$. For numerical convenience, we represent its energy distribution with a narrow Gaussian distribution shown in Figure 4(e)-(h) with thin solid dark red line. Different rows show distributions for different values of $E_{0} / m_{e} c^{2}: 10^{6}, 10^{7}, 1.6 \times 10^{9}$ (fiducial value), and $10^{10}$. We then evolve the system of equations (37)-(38) numerically, as described in Section 3.2, and show subsequent distributions in Figure 4 with colored lines (see the legend).

We adopt as our fiducial model pair cascade for M87 the case when $E_{0}=1.6 \times 10^{9} m_{e} c^{2}$, shown in Figure $4(\mathrm{~g})$. The electron cools (see Equation 33) due to the upscattering of a seed photon into a $\gamma$-ray photon, which in turn pair produces on an additional seed photon (see Equation 34). These two contributions to lepton energy distribution are clearly visible in Figure 4(h): after the first scattering, i.e., in generation 1, the IC-cooled electron distribution is a power-law extending from $\gamma_{\text {lep }} \approx 0.5 \epsilon_{\gamma \text {,min }}=6 \times 10^{5}$ to $\gamma_{\text {lep }}=2 \times 10^{8}$ and the new pairs form a Gaussian distribution centered around $\gamma_{\text {lep }} \approx 7 \times 10^{8}$. Note that the lower-energy cutoff in the power-law emerges because the seed photon bath is transparent to gamma-rays with energy less than $\epsilon_{\gamma, \min }$.

Numerically-obtained values of density enhancement vs. generation number are shown in Figure 5(a), for different values of $E_{0}$. It is clear that the density enhancement saturates around generation $N_{\text {gen }} \gtrsim$ few $\times 10$. The numerical result for density enhancement in our fiducial model of M87, $n_{\infty} / n_{g}=$ 640 , is shown with long-dashed magenta line and is in excellent agreement with the analytic expectation, discussed above and given by Equation (28), $n_{\infty} / n_{g} \approx 670$. This yields a jet lepton density near the stagnation surface of

$$
n_{\infty} \approx 15 \mathrm{~cm}^{-3} \text {. }
$$

In the absence of nonlinear plasma phenomena, these energic pairs will inverse-Compton cool over roughly a single jet scale height, i.e., within a distance $r$, at which point they will have undergone large-angle deflections. These leptons, now misaligned with the background magnetic field, will subsequently synchrotron cool and isotropize. The rate of this cooling depends on the microscopic Lorentz factors and becomes comparable to the outflow timescale only when $\gamma \approx 10^{2-3}$ on scales of the gap height. Thus the resulting population is in excellent agreement with that required to produce the observed sub-mm emission.

As is also clear from Figure 5(a), higher initial energy leads to a higher density enhancement. Similarly, Figure 5(b) shows that the total energy and the energy in leptons are higher for higher $E_{0}$. The flatness of the solid lines in Figure 5(b) demon- 

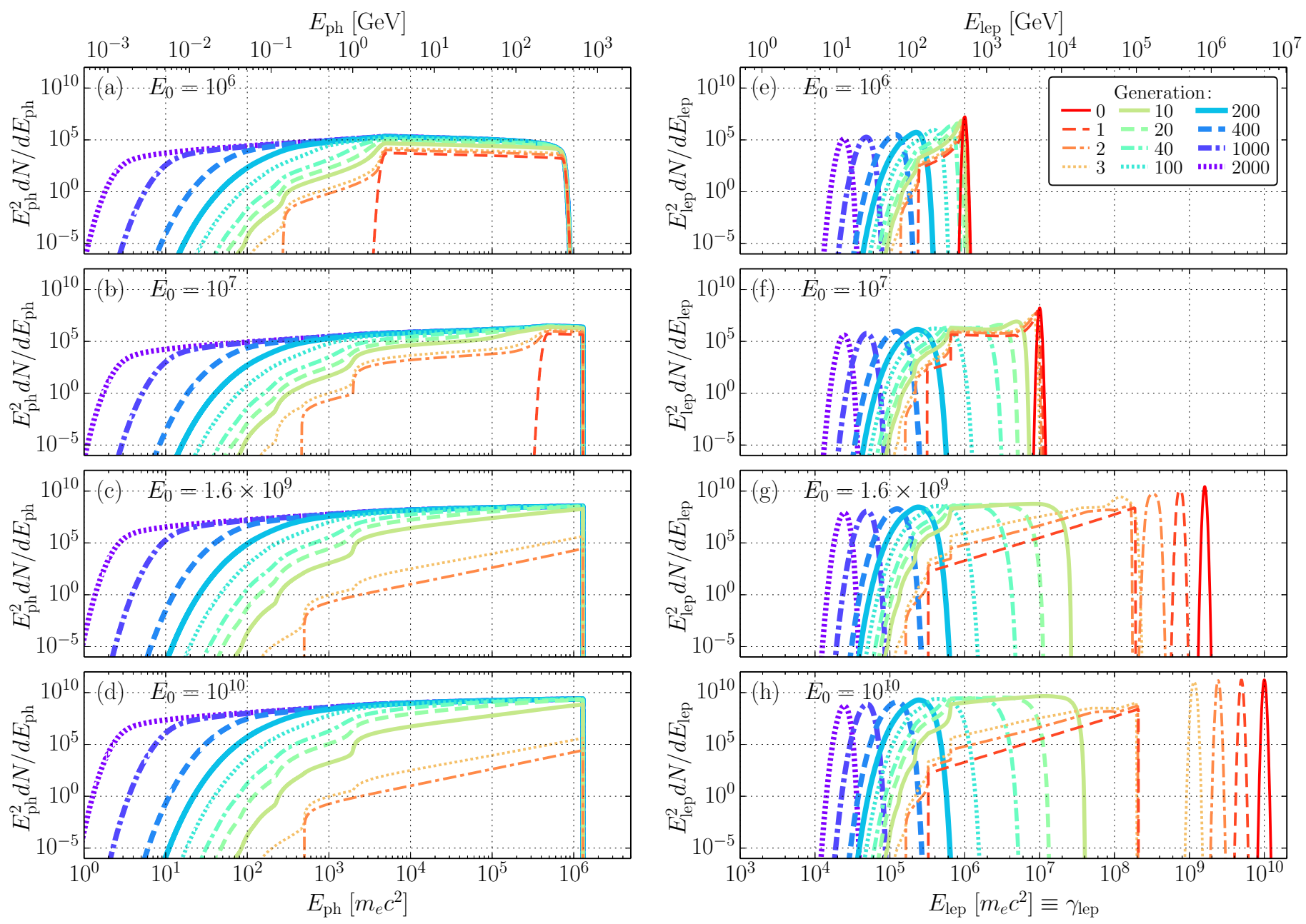

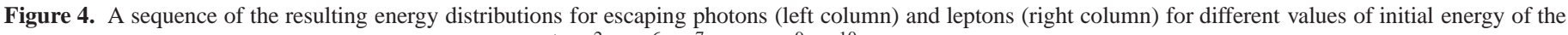

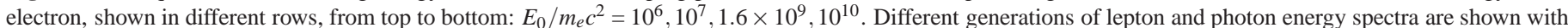

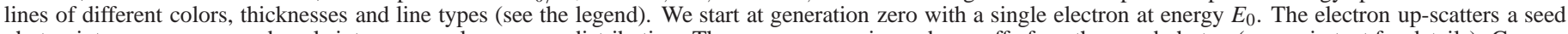

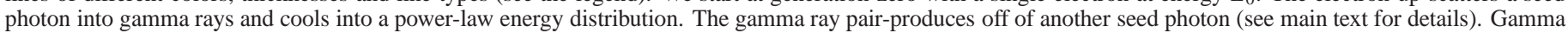

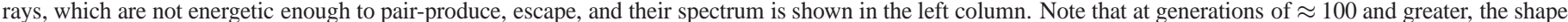

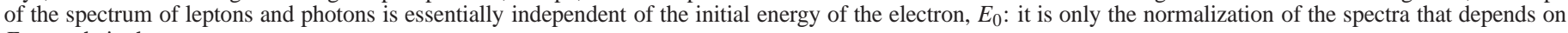
$E_{0}$, not their shape.

strates that the total energy of the cascade (carried by leptons and photons) is conserved at high accuracy by our numerical scheme. Note that as seen in Figure 4, there exist asymptotic properties of the pair cascade that are independent of $E_{0}$. For example, it is explicitly apparent in Figure 5(c) that the average lepton Lorentz factor, $\left\langle\gamma_{\text {lep }}\right\rangle$, is independent of $E_{0}$ for $N_{\text {gen }} \gtrsim 100$ and is controlled completely by $\tau_{\gamma \gamma}$.

We investigated the dependence of density enhancement factor on key parameters of our model, and the results are shown in Figure 6. The blue dots connected by solid lines show the numerical results; they are in excellent agreement with the analytical scaling given by Equation (28). The density enhancement is most sensitive to the initial electron energy, $E_{0}$ : $N_{\text {lep, } \infty} \propto E_{0}$ [see Figure 6(a)], and the gamma-ray energy below which the seed photon bath is optically-thin to pair production, $\epsilon_{\gamma, \min }: N_{\mathrm{lep}, \infty} \propto \epsilon_{\gamma, \min }^{\alpha /(2 \alpha-1)} \approx \epsilon_{\gamma, \min }^{0.86}$ (see Figure 6(c)). However, if we now recall that both $E_{0}=\gamma_{\max , \text { IC }} m_{e} c^{2}$ and $\epsilon_{\gamma \text {,min }} \propto \gamma_{\max \text {,IC }}$

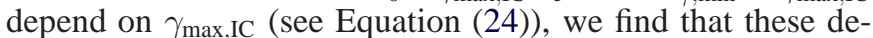
pendencies nearly cancel out, and that density enhancement is most sensitive to the density of seed photons $n_{s}$, or source lu- minosity:

$$
\frac{n_{\infty}}{n_{g}} \propto\left(\gamma_{\max , \mathrm{IC}} \epsilon_{b}\right)^{(\alpha-1) /(2 \alpha-1)} n_{s}^{1 /(2 \alpha-1)} \approx\left(\gamma_{\max , \mathrm{IC}} \epsilon_{b}\right)^{0.14} n_{s}^{0.71}
$$

Importantly, the numerical results are insensitive to the upper energy cutoff of the seed photon spectrum, as shown in Figure 6(d).

We ensured that our simulation results are numerically converged. For this, we varied the number of energy bins for more than 3 orders of magnitude and computed the error relative to the highest-resolution simulation carried out using $2 \times 10^{5}$ energy bins. As seen in Figure 7, the simulation results converge at first order with increasing energy resolution. For the simulations described here, we employed a fiducial resolution of $10^{4}$ energy bins, for which the relative error is less than 0.3 per cent in all quantities.

The asymptotic Lorentz factor after the pair cascade saturates, set by when the inverse-Compton cooling length is comparable to the jet scale height, is roughly $10^{4}$. This implies direct synchrotron emission up to $\approx 10-100 \mathrm{eV}$, well into the 

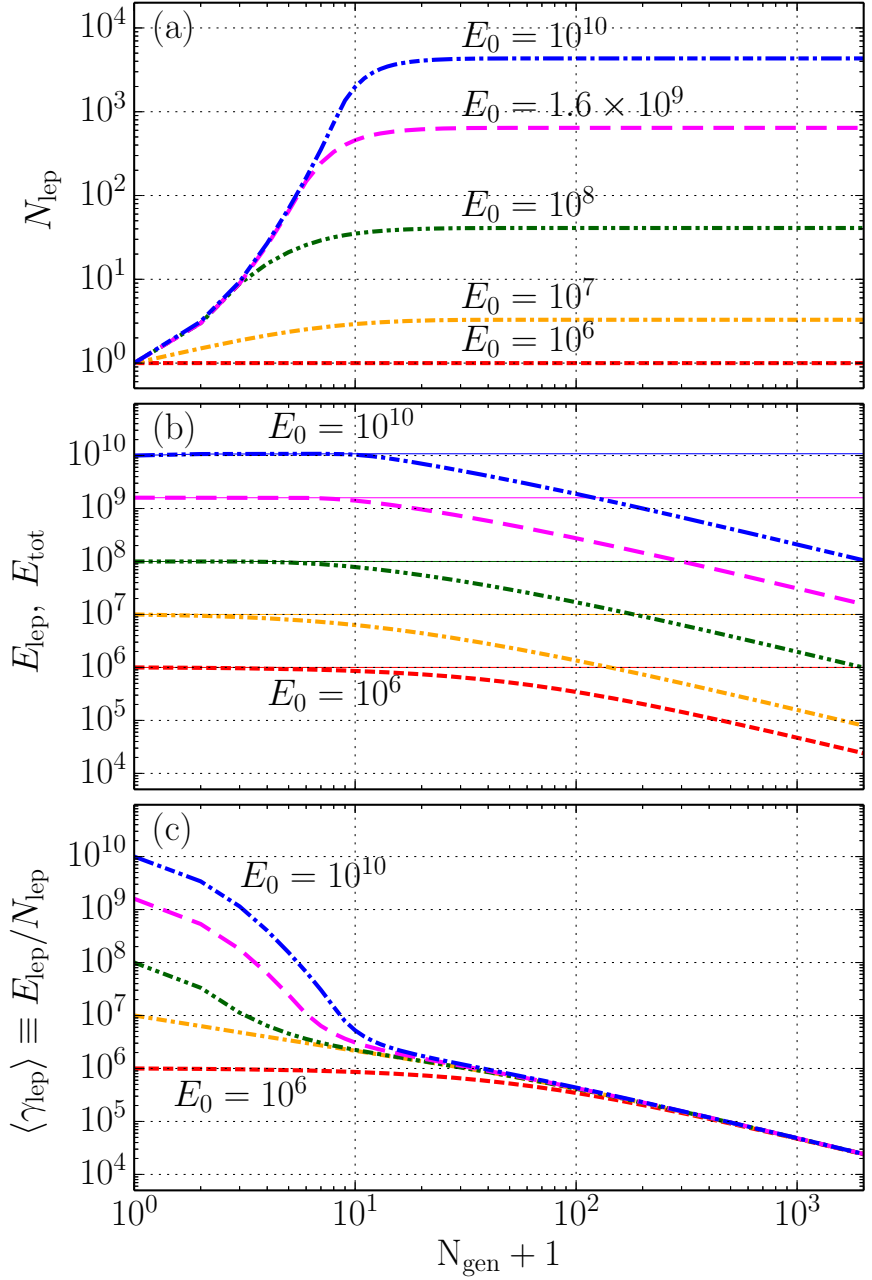

Figure 5. Evolution of various quantities in pair cascade with generation for different values of initial lepton energy, $E_{0}$, which is measured in units of $m_{e} c^{2}$. Panel (a) shows the dependence on the generation number of density enhancement, $N_{\text {lep }} \equiv n_{\infty} / n_{g}$. Panel (b) shows the dependence of the total energy in leptons and photons with colored solid lines and the lepton total energy, $E_{\text {lep }}$, with other lines, for different values of initial lepton energy, $E_{0}$. Panel (c) shows the evolution with the generation number of the average lepton Lorentz factor, $\left\langle\gamma_{\text {lep }}\right\rangle$.

ultraviolet.

\subsection{Direct Inverse Compton Signal}

The direct inverse Compton signal from the stagnation surface itself should be visible up to $\epsilon_{\mathrm{VHEGR}} \approx 640 \mathrm{GeV}$, above which it will be absorbed by the pair cascade. As is clear from Figure 4, the direct IC signal from the cascade has a flat energy spectrum in $E^{2} d N / d E$, with every decade in energy carrying approximately the same amount of energy. This is consistent with the analytical expectation given in Appendix C. Thus, it is possible that the observed very-high energy gamma-ray emission is associated with the stagnation surface (see, e.g., Levinson \& Rieger 2011). Emission in the band pass of Fermi/LAT, around $\epsilon_{\gamma} \approx 10 \mathrm{GeV}$, is mostly due to Compton up-scattering of seed photons near the peak of the SED, $\epsilon_{s} \approx 1.2 \mathrm{meV}$, by electrons with Lorentz factors $\gamma \approx\left(\epsilon_{\gamma} / 2 \epsilon_{S}\right)^{1 / 2}=2 \times 10^{6}$, which is comparable to $\gamma_{\gamma \gamma}$. The associated power per lepton is

$$
P_{10 \mathrm{GeV}} \approx 4 \sigma_{T} u_{s} c \gamma^{2} / 3
$$
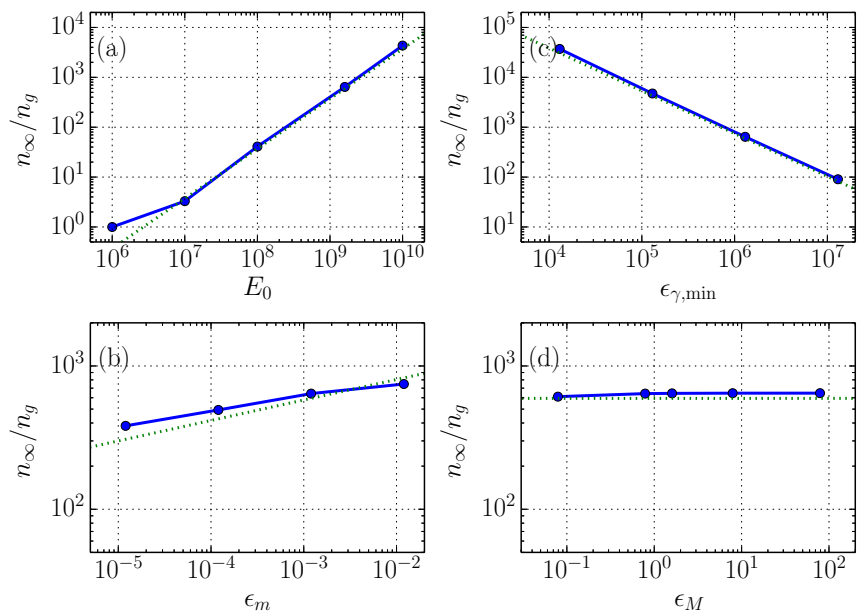

Figure 6. Dependence of density enhancement factor, $n_{\infty} / n_{g}$, on the parameters of the cascade. Connected blue dots show simulation results and the green dotted lines show the analytical expression given by Equation (28). Panel (a) shows the dependence of density enhancement factor, $n_{\infty} / n_{g}$ on the initial energy $E_{0}$ of the lepton, panel (b) on the lower energy cutoff in the seed photon distribution $\epsilon_{m}$, panel (c) on the minimum energy of gamma-rays able to pair produce $\epsilon_{\gamma \text { min }}$ (see Equation 24), and panel (d) on the upper energy cutoff in the seed photon distribution. The agreement between the model and the simulation is better than $30 \%$.

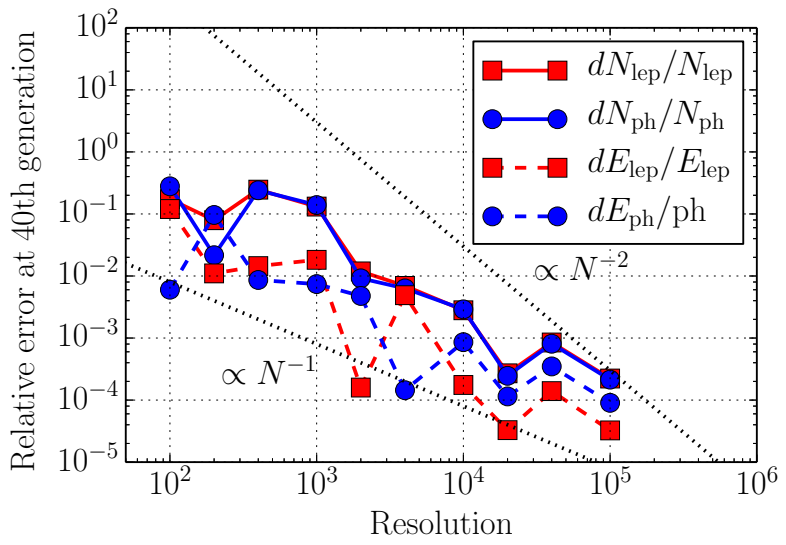

Figure 7. Dependence of fractional error on resolution in our fiducial model (evaluated at 40th lepton generation) for various quantities: lepton number, $N_{\text {lep }}$, emitted photon number, $N_{\text {ph }}$, lepton energy, $E_{\text {lep }}$, emitted photon energy, $E_{\mathrm{ph}}$. The error is computed relative to a high-resolution model with $2 \times 10^{5}$ spectral energy bins, and exhibits linear convergence. At our fiducial resolution of $10^{4}$ energy bins, the relative error is less than 0.3 per cent in all quantities.

The highly relativistic nature of the lepton distribution produces a large relativistic aberration, beaming the up-scattered gamma rays within a cone with an opening angle $1 / \gamma$ around the original electron momentum vector, and thus concentrating the emission in a solid angle of $\Omega_{\gamma}=\pi / \gamma^{2}$. However, it also limits the region of the stagnation surface that can be viewed to an area $\Omega_{\gamma} r^{2}$. Finally, inverse-Compton cooling also sets a length scale over which we expect to find particles that can produce sufficiently energetic up-scattered gamma rays, $\ell_{\mathrm{IC}, 10 \mathrm{GeV}}$ typically much smaller than $r_{\mathrm{g}}$, and here roughly $0.05 r_{\mathrm{g}}$ (see Equation 6). Thus, the typical anticipated $10 \mathrm{GeV}$ flux produced near the stagnation surface is independent of the beaming, assuming that the viewing angle is within the jet opening 
angle:

$$
\begin{aligned}
\left.F_{\epsilon}\right|_{10 \mathrm{GeV}} & \approx \frac{P_{10 \mathrm{Gev}}}{\Omega_{\gamma}} n_{\infty} \frac{\Omega_{\gamma} R_{j}^{2}}{D^{2}} \ell_{\mathrm{IC}, 10 \mathrm{GeV}}=\gamma m c^{3} n_{\infty} \frac{\pi R_{j}^{2}}{D^{2}} \\
& =7.1 \times 10^{-9} \mathrm{erg} \mathrm{cm}^{-2} \mathrm{~s}^{-1} .
\end{aligned}
$$

In principle, all leptons with Lorentz factors above $\gamma$ will contribute to the energy flux. In practice, this increases the flux near $10 \mathrm{GeV}$ by a factor of less than two (see Appendix C), to

$$
\begin{aligned}
\left.\epsilon F_{\epsilon}\right|_{10 \mathrm{GeV}} & \approx \frac{12(\alpha-1)}{2 \alpha-1} \gamma m c^{3} n_{\infty} \frac{\pi R_{j}^{2}}{D^{2}} \\
& =1.2 \times 10^{-8} \mathrm{erg} \mathrm{cm}^{-2} \mathrm{~s}^{-1} .
\end{aligned}
$$

This is considerably larger than the $(7.5 \pm 5.9) \times$ $10^{-12} \mathrm{erg} \mathrm{cm}^{-2} \mathrm{~s}^{-1}$ reported for M87 by Fermi in the first Fermi/LAT catalog of $>10 \mathrm{GeV}$ sources (1FHL, Ackermann et al. 2013). It is, however, consistent with the distanceadjusted fluxes of other gamma-ray bright blazars in the Fermi sample (see, e.g., Ackermann et al. 2011).

The disparity is almost certainly due to the high degree of beaming anticipated. At $10 \mathrm{GeV}$, the high energy emission is beamed within 0.1 " of the original electron direction. Beyond this angle, the energy of the up-scattered photons drops dramatically; at $10^{\circ}$ the typical up-scattered photon energy is only roughly 70 times larger than that of the original seed photon, well below the energies of interest. Thus, the gammaray emission from the stagnation surface would be expected to drop precipitously outside of the jet half-opening angle. This is a strong function of height, scaling $\propto z^{-1 / 2}$ near the black hole, though at our fiducial height of the stagnation surface this is $\theta_{j} \equiv R / z \approx 18^{\circ}$. Because the jet is collimating the tangent to the field lines, which correspond to the range of angles over which the relativistic leptons are directed and subsequent gamma rays emitted, makes a considerably smaller angle with the jet axis, $\theta_{b} \approx 0.5 \theta_{j}=9^{\circ}$ for a parabolic jet. Both of these are well within the inclination implied by radio observations of $25^{\circ}$, suggesting that it in this case it would be rare to find substantial gamma-ray emission in M87.

\subsection{Total power of the cascade}

Using Equation (21), we obtain the total power dissipated by the cascade,

$$
L_{\text {cascade }} \approx 10^{43} \mathrm{erg} \mathrm{s}^{-1},
$$

corresponding to roughly $10 \%$ of the total jet power, $L_{\text {jet }} \approx$ $10^{44} \mathrm{ergs}^{-1}$. Thus, most of the jet power flows out in the form of Poynting flux and only $10 \%$ of it gets converted into particle energy at the stagnation surface and the cascade. Most of this power is radiated in the form of gamma-rays and is not observable due to beaming away from our line of sight, as we discussed above. A small fraction of this power,

$$
\begin{aligned}
P_{\infty} & =\frac{\gamma_{\infty} n_{\infty}}{\gamma_{\text {max }, \text { IC }} n_{g}} L_{\text {cascade }}=\frac{\gamma_{\infty}}{\gamma_{\gamma \gamma}} L_{\text {cascade }} \\
& \approx 4 \times 10^{-3} L_{\text {cascade }}=5 \times 10^{40} \mathrm{erg} \mathrm{s}^{-1},
\end{aligned}
$$

is carried asymptotically by the cascade and will be radiated via synchrotron emission. This is roughly a factor of 20 smaller than the isotropic-equivalent luminosity, consistent with the beaming correction inferred from detailed source modeling Broderick \& Loeb (2009). ${ }^{6}$ This is not surprising since the

\footnotetext{
${ }^{6}$ Note that since the angular size of the source is fixed in the estimates of the seed photon densities, the beaming correction does not affect $u_{s}$.
}

asymptotic values of density and Lorentz factor produced by the cascade, $n_{\infty}$ and $\gamma_{\infty}$, are consistent with those necessary to explain M87's radio emission, as discussed in §6.

We note that in addition to total energy budget our model has the potential to address an important morphological feature of M87 jet that is pronounced in radio images at a frequency of $43 \mathrm{GHz}$ (Walker et al. 2008): edge-brightening of the jet. The electric field in the gap, given by Equation (1), increases away from the rotational axis. This leads to density enhancement peaked toward the edge of the jet (see Equations 8 and 61) and therefore has the potential to lead to stronger jet emission near the jet edges. More detailed study of this issue is warranted.

\section{APPLICATION TO SGR A*}

A second obvious application is to the putative jet in Sgr A*, the black hole at the center of the Milky Way. Unlike M87, there is no obvious radio jet in $\mathrm{Sgr} \mathrm{A}^{*}$, making the task of constraining its properties somewhat less well defined. However, there is some recent evidence for a collimated outflow containing a non-relativistic population of leptons ( $\mathrm{Li}$ et al. 2013). In this case the putative jet lights up on parsec scales as a result of intersecting, and shock heating upon, a stream of gas in Sgr A*'s vicinity. Again, we will first review some relevant parameters for $\mathrm{Sgr} \mathrm{A}^{*}$ and then assess the implications of the particle acceleration model presented in Section 2.

This is complicated by the current uncertainty regarding the dynamics and morphology of the region responsible for the observed sub-mm emission. This uncertainty manifests itself primarily in the assumed magnetic field strength. In sections 5.15.3 we will assess in detail the implications for a putative jet assuming that the observed emission arises primarily in a RIAF. In section 5.5 we consider the possibility of a self-consistent solution in which the jet itself is responsible for the sub-mm emission.

\subsection{Sgr A* Source Parameters}

The mass of and distance to Sgr $\mathrm{A}^{*}$ are the best known of any black hole candidate, obtained from observations of the orbits of individual stars, yielding $M=4.3 \pm 0.4 \times 10^{6} M_{\odot}$ and $8.3 \pm 0.4 \mathrm{kpc}$, respectively (Ghez et al. 2008; Gillessen et al. 2009). As with M87, the SED peaks near $1 \mathrm{~mm}$, where the typical observed flux is roughly $3 \mathrm{Jy}$. However, the isotropic-equivalent luminosity is much lower, roughly $L \approx 10^{36} \mathrm{erg} \mathrm{s}^{-1} \approx 10^{-9} L_{\text {Edd }}$. Hence, even in Eddington units, Sgr $\mathrm{A}^{*}$ is considerably more underluminous than M87, for which $L \approx 10^{42} \mathrm{erg} \mathrm{s}^{-1} \approx 10^{-6} L_{\text {Edd }}$. Here we collect various parameter estimates relevant for the putative jet launching region near Sgr A*.

We will find that Sgr A* and M87 have roughly similar photon energy densities, the frequency of the spectral peak, and magnetic field strengths. This leads to comparable (to within a factor of few) physical (in cm) gap thicknesses in both cases. However, the mass of Sgr A* black hole is about 1500 smaller than in M87. This means that in SgrA*, the gap thickness is comparable to large-scale size of the system, and this leads to the suppression of the cascade.

\subsubsection{Jet Velocity and Orientation}

In the absence of an obvious jet, there are no direct measurements of a putative jet velocity. In contrast, if the jet feature reported in Li et al. (2013) is real, it is necessarily oriented at a large angle relative to the line of sight. Here we will assume a similar, moderate Lorentz factor for the jet $(\Gamma \approx 2.3)$ and an 
inclination angle of $90^{\circ}$, where necessary, consistent with the assumed parameters in Li et al. (2013).

\subsubsection{Magnetic Field Strength Estimates \& Synchrotron Cooling Times}

The detection of polarized emission at mm-wavelengths, and thus the measurement of the Faraday rotation measure of $\approx 6 \times$ $10^{5} \mathrm{rad} \mathrm{m}^{-2}$, produces an estimate for the accretion rate of $\dot{M}=$ $2 \times 10^{-9} M_{\odot} \mathrm{yr}^{-1}-2 \times 10^{-7} M_{\odot} \mathrm{yr}^{-1}$, depending on the details of the accretion model assumed (Agol 2000; Marrone et al. 2007). Following equations (41)-(42) gives a corresponding estimate of the possible confined jet magnetic field of up to

$$
B \approx \sqrt{\frac{2 \beta c \dot{M}}{r_{\mathrm{g}}{ }^{2}}} \approx 10^{2-3} \mathrm{G} .
$$

In both cases, the accretion power, ranging from $10^{38}-10^{40} \mathrm{erg} \mathrm{s}^{-1}$, exceeds the potential jet power by two orders of magnitude, and imply the need for low radiative efficiencies, i.e., $\eta_{d} \approx 0.01-10^{-4}$. These field strengths are similar to those inferred from spectral fitting (e.g., Yuan et al. 2003; Broderick \& Loeb 2006). For concreteness we will assume the lower field strength, corresponding to the comparatively higher radiative efficiency, i.e., we will assume $100 \mathrm{G}$ on the horizon and thus roughly $10 \mathrm{G}$ near the stagnation surface. As a consequence, the synchrotron cooling timescale is similar to that of M87:

$$
t_{\text {sync }} \approx 4.0 \times 10^{5} B_{1}^{-3 / 2} \nu_{11.5}^{-1 / 2} \mathrm{~s} .
$$

Since the gravitational radius of $\mathrm{Sgr} \mathrm{A}^{*}$ is much smaller, so is the apparent outflow timescale, comparable to

$$
t_{\text {outflow }} \approx 2.4 \times 10^{2}\left(\frac{r}{10 r_{\mathrm{g}}}\right) \mathrm{s},
$$

and thus for Sgr A* synchrotron cooling is completely irrelevant to the evolution nonthermal particle population on horizon scales.

\subsubsection{Seed Photon Density Estimates}

Unlike M87, Sgr A* exhibits a clear sub-mm bump, often modeled as a thermal disk component (Yuan et al. 2003), though also potentially produced by the jet component associated with the jet launching region (Falcke \& Markoff 2000; Yuan et al. 2002; Mościbrodzka \& Falcke 2013). Nevertheless, above $1 \mathrm{~mm}$ the SED is well modeled by a power law with $\alpha \approx 1.25$ (see Figure 8 ):

$$
\alpha \approx \begin{cases}0 & \nu<300 \mathrm{GHz} \\ 1.25 & \nu \geq 300 \mathrm{GHz} .\end{cases}
$$

Furthermore, short-timescale variability from the mm through the infrared imply that this emission arises near the black hole, and thus may be identified with the soft seed photons relevant for particle production and acceleration at the putative jet stagnation surface.

The implied seed photon energy density above $1 \mathrm{~mm}$ is

$$
u_{s} \approx 1.5 \times 10^{-2} \mathrm{erg} \mathrm{cm}^{-3},
$$

which is comparable to that implied in M87 (see Equation 50). We will find, however, that the relevant seed photon population lies above $10^{12} \mathrm{~Hz}$ (wavelengths shorter than $0.26 \mathrm{~mm}$ ), for which the energy density is reduced by roughly a factor of 5 .

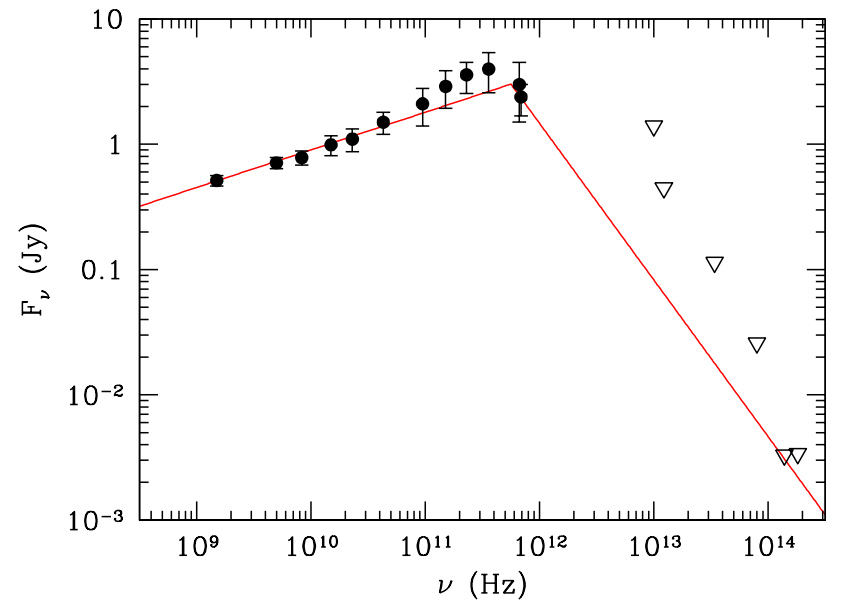

Figure 8. Spectral energy distribution for Sagittarius A* from the radio to the optical, collected from Yuan et al. (2004, and references therein) and Marrone (2006). Filled circles and open triangles correspond to flux measurements in which the source is resolved and unresolved, respectively; we take the filled circles as directly indicative of the near-horizon seed photon distribution and the open triangles as upper limits. Errorbars indicate variability, not intrinsic measurement uncertainty. For comparison, a simple broken power-law with high-frequency spectral index $\alpha=1.25$, as employed in the text, is shown for reference.

As we will see below, this leads to about a factor of 3 increase in the physical thickness of the gap (in $\mathrm{cm}$ ) in comparison to M87. This turns out to lead a qualitative change in the cascade operation: since Sgr A* black hole mass is $\sim 1500$ times smaller than in M87, Sgr A* gap thickness comes out to be comparable to the size of black hole, and this suppresses the cascade.

\subsubsection{Implied Nonthermal Particle Properties}

The roughly power-law, declining SED of Sgr A* in the sub$\mathrm{mm}$ again justifies assuming the emission is well characterized by optically thin emission from a soft population of nonthermal electrons. In this case, $d n / d \gamma \propto \gamma^{-3.5}$, quite similar to M87. With a source size of $37 \mu$ as, equating the expression in Equation (51) to the observed millimeter flux of $3 \mathrm{Jy}$ yields a nonthermal particle density estimate of

$$
n \approx 10^{6} \mathrm{~cm}^{-3} \text {. }
$$

This is much higher than for M87 as a direct result of the much smaller mass, and therefore more compact emission region.

Given the previous magnetic field estimate, the typical Lorentz factors are expected to be of order $10^{2}$. While the infrared emission requires considerably larger $\gamma$ 's, it is also highly variable and thus potentially associated with additional dissipative events.

\subsection{Particle Acceleration at the Stagnation Surface}

As might be expected given the similarities in the soft photon densities, the inverse-Compton limited Lorentz factor is about a factor of 2 smaller than that found in M87 (see Equation 3; see Equation 53 for M87 value):

$$
\gamma_{\max } \approx 7.6 \times 10^{8}
$$

The length scale over which this is attained is (see Equation 6)

$$
\ell_{\mathrm{IC}} \approx 8.4 \times 10^{10} \mathrm{~cm} \approx 0.13 r_{\mathrm{g}}
$$


This value in $\mathrm{cm}$ is larger by about a factor of 2 and in units of gravitational radius by about a factor of 2300 than the M87 value (see Equation 55). As a consequence of the moderately lower $\gamma_{\max }$ the threshold energy for seed photons which are able to pair produce on the subsequently up-scattered inverseCompton gamma is moderately higher,

$$
\epsilon_{\mathrm{th}}=2.7 \mathrm{meV} \Rightarrow \lambda_{\text {th }} \approx 0.46 \mathrm{~mm},
$$

which is pushing into the far-infrared. Hence, unlike in M87, for Sgr A* the soft photons responsible for pair production in the gap are significantly above the spectral break.

Due to the larger $\epsilon_{\mathrm{th}}$, the corresponding mean free path to pair production is comparatively large (see Equation 15),

$$
\ell_{\gamma \gamma} \approx 6.6 \times 10^{12} \mathrm{~cm} \approx 10 r_{\mathrm{g}} .
$$

This value exceeds that for M87 by a factor of $\simeq 4$ in $\mathrm{cm}$ and in units of $r_{g}$ by a factor of 5900 (see Equation 56). This implies that at most, the gap pair catastrophe is limited to a single generation. This suggests that the global jet dynamics, will play a significant role in the determining the structure of the gap. Ignoring this potential complication, the equilibrium gap thickness is of order (see Equation 17; see Equation 57 for M87 value)

$$
\Delta \approx 1.1 \times 10^{12} \mathrm{~cm} \approx 1.7 r_{\mathrm{g}},
$$

itself larger than a gravitational radius (though given the wide disparity between $\ell_{\text {IC }}$ and $\ell_{\gamma \gamma}$ this may be highly variable). The associated density at the top of the gap is (see Equation 18)

$$
n_{\mathrm{g}} \approx 2.5 \times 10^{-3} \mathrm{~cm}^{-3},
$$

about an order of magnitude smaller than for M87 (Equation 58).

In summary, the gap densities for Sgr A* (Equation 78) and M87 (Equation 58) are within an order of magnitude of each other because of the similarity of seed photon densities, magnetic field strengths, and the peak frequency of the spectrum. The order of magnitude smaller density in Sgr A* comes from a factor of few smaller values of magnetic field strength and above threshold photon density for Sgr A*, falling well short of the $n_{\infty} \simeq 10^{6} \mathrm{~cm}^{-3}$ inferred from radio observations (see Sec. 5.1.4).

\subsection{Post-Gap Cascade}

Since $\ell_{\gamma \gamma}$ is much larger than the system scale height at the gap, i.e., $\ell_{\gamma \gamma} \gg r \approx 10 r_{\mathrm{g}}$, there is effectively no post-gap cascade. This is because for compact emission regions the pairproduction optical depth falls significantly at large radii for two reasons. First, the seed photon density drops $\propto r^{-2}$ for sufficiently large $r$. Second, the direction of propagation of the seed photons and gamma rays become increasingly aligned as they travel far from their origin, reducing their center-of-mass energy and increasing the energy threshold for pair production $\propto r^{2}$. Hence a gamma ray that fails to annihilate in the first mean free path is unlikely to ever do so.

Thus, $n_{\infty} \approx n_{\mathrm{g}} \approx 2.5 \times 10^{-3} \mathrm{~cm}^{-3}$, implying that in this case it is likely that Sgr A* emission is likely coming from the accretion disk or from the disk outflow just outside of the highly magnetized jets (e.g., Mościbrodzka \& Falcke 2013). If a substantial fraction of Sgr A* arises from jet emission (but note that this does not have to be the case: in fact, SgrA* might not have any jet at all), some other particle loading and acceleration mechanisms must be occurring, such as gas entrainment from the accretion disk and the acceleration of electrons via reconnection and/or shocks.

\subsection{Gamma-Ray Emission}

According to Equations (20) and (21), the pair cascade luminosity scales as $\propto M^{2} n_{g} \gamma_{\text {max.IC. Since black hole mass is }}$ smaller by 3 orders of magnitude, density is smaller by an order of magnitude, and $\gamma_{\max , I C}$ is smaller by a factor of two, the pair cascade luminosity is roughly $\sim 7$ orders of magnitude smaller than in M87 (Equation 65), or $\approx 3 \times 10^{35} \mathrm{erg} \mathrm{s}^{-1}$. Since $\gamma_{\infty} \approx 10^{7}$ the overwhelming majority of this is deposited in gamma rays beamed along the jet with typical energies of $\gamma_{\max } m_{e} c^{2} \approx 400 \mathrm{TeV}$.

Based on the overall gamma-ray luminosity it is tempting to relate this to the power in the $1 \mathrm{GeV}-100 \mathrm{GeV}$ gamma-ray $10 \mathrm{kpc}$-scale jet features found by Su \& Finkbeiner (2012). Such an identification, however, requires at least two additional mechanisms. First, the gamma rays must be reprocessed down in energy by between three and five orders of magnitude. Second, the emission must be scattered in a distributed fashion along the jet to produce the observed extended feature. Accordingly, we leave the discussion of potential signatures of gamma-ray emission from $\mathrm{Sgr}$ A*'s putative jet for future work.

\subsection{Self-Consistent Jet Solutions}

We finish our discussion of $\operatorname{Sgr} \mathrm{A}^{*}$ with a consideration of potentially self-consistent jet solutions. While it has been found above that a jet confined by an observationally motivated RIAF models is expected to produce little intrinsic emission, this need not be the case if the RIAF picture is relaxed.

Increasing the magnetic field increases the efficiency of the inverse-Compton pair catastrophe. For $B \gtrsim 200 \mathrm{G} \ell_{\gamma \gamma}$ becomes small in comparison to the gap height, and thus multiple generations of pairs can be sustained resulting in a well-defined inverse-Compton pair catastrophe. Simultaneously, the number of pairs required to reproduce the observed sub-mm emission decreases. For $B \approx 10^{4} \mathrm{G}$ the two are approximately equal, suggesting the existence of a self-consistent jet model similar to that found for M87. As with weaker fields synchrotron cooling may continue to be ignored (this is true for $B \lesssim 1.5 \times 10^{4} \mathrm{G}$ ).

However, note that $10^{4} \mathrm{G}$ would be much larger than even optimistic estimates for the magnetic field strength in Sgr A*. The implied jet power would be roughly $L_{\mathrm{EM}} \gtrsim 10^{41} \mathrm{erg} \mathrm{s}^{-1}$, five orders of magnitude larger than Sgr A*'s bolometric luminosity. While such large luminosities have been implicated in Sgr A*'s past (e.g., the Fermi bubbles Su et al. 2010), it is difficult to imagine sufficiently low radiative efficiencies to hide such jet power in the present epoch.

Perhaps more problematic is the need to confine such a large magnetic flux. This is ostensibly done via an accretion flow, which implies a mass accretion rate of

$$
\dot{M} \approx \frac{B^{2} r_{\mathrm{g}}^{2}}{2 \beta c} \gtrsim 10^{-5} M_{\odot} \mathrm{yr}^{-1},
$$

comparable to the Bondi rate for Sgr A*. As discussed in Section 5.1.2, such high accretion rates are already excluded by polarimetric observations. Moreover, the corresponding radiative efficiency of the accretion flow would need to be exceedingly small: $\eta_{d} \lesssim 10^{-6}$, much lower than the already small efficiencies implicated in RIAF models.

For these reasons we consider a self-consistent jet model driven by a gap-powered inverse-Compton pair catastrophe to 
be highly disfavored as an explanation of Sgr A*'s current sub-mm emission. Nevertheless, this may not be the case for Sgr A*'s recent past, where the luminosity of Sgr A* has been inferred to be considerably higher.

\section{DISCUSSION}

\subsection{Source of seed photons}

Our model relies on the presence of seed photons with energies $\epsilon_{s} \approx 1 \mathrm{meV}$, or wavelengths $\lambda \approx 1 \mathrm{~mm}$, to sustain the cascade. The spectra of Sgr A* and M87 above the peak of the SED give us an estimate of the seed photon density at the source. However, so far we have been agnostic about the origin of these photons: whereas in the case of Sgr A* they could plausibly only come from the accretion disk, in M87 they could come from the disk, the jet, or both (see, e.g., Broderick \& Loeb 2009).

In our model of M87, the synchrotron luminosity of the jet, which is lit up by the leptons accelerated in the cascade, naturally explains the SED of M87 above its peak at $\epsilon_{s} \gtrsim 1 \mathrm{meV}$. This is because the pair cascade catastrophe naturally leads to pair density of $n_{\infty} \approx 15 \mathrm{~cm}^{-3}$ and Lorentz factor $\gamma_{\infty} \approx 10^{4}$ (see $\$ 4.3$ and $\$ 4.5$ ): both of these values are comparable to the those required to account for M87's radio emission above the peak of the SED (Broderick \& Loeb 2009). This means that in principle no external source of seed photons is required: the cascade is self-sustaining, similar to polar cascades in pulsar magnetospheres (see, e.g., Timokhin \& Arons 2013). That is, given a stray seed photon (e.g., from an accretion disk), the cascade fires up and produces energetic electrons that then cool and produce sub-mm photons that can serve as seed photons for the cascade.

\subsection{Comparison to other work}

An alternative process of pair production in jets is annihilation of gamma-rays from the disk (Levinson \& Rieger 2011; Mościbrodzka et al. 2011). In this scenario, a sub-mm photon, emitted by the disk, undergoes a sequence inverse Compton scattering events with disk's own hot electrons. These interactions upscatter the photon to gamma-ray energies, and eventually above pair production threshold. Collisions of these gamma-rays in the funnel mass-load the jet with pairs.

In order for this process to lead to an interestingly large number density of pairs (larger than the Goldreich-Julian density, $n_{\mathrm{GJ}}$ ), the following two conditions must be met: (i) the mmemission of the disk must be compact and marginally optically thick to Thompson scattering; (ii) disk electrons must be very hot, so that on average no more than $\approx 1.5$ Compton scatterings are needed to reach pair-production threshold. To satisfy these requirements, disk radiative efficiency is assumed to be high, $\eta_{d} \approx 0.3$ (Mościbrodzka et al. 2011).

In contrast, our model allows for standard interpretation of a radiatively inefficient accretion flow in $\mathrm{M} 87, \eta_{d} \ll 1$, which we believe is a more natural assumption. The seed photons around the SED peak are produced in the jet, outside of the disk proper, and thus they have a lower probability of being up-scattered to gamma-ray energies by disk electrons. Both of these factors combined render ineffective the process of jet pair loading due to $\gamma-\gamma$ collisions in the context of our model.

Levinson \& Rieger (2011) considered pair cascade at the stagnation surface of black hole magnetosphere and focused on the gamma-ray emission from the resulting leptons. In our work we take a broader approach and construct quantitative 1D numerical simulations of the post-cascade evolution. We con- sider both the direct gamma-ray signal as well the synchrotron emission of the leptons produced in the cascade. In fact, we argue that the seed photons are produced in the cascade itself and not the accretion flow. We use the observed spectrum of M87 to determine the seed photon spectrum and eliminate the sensitivity of the model to the uncertain details of the accretion flow.

\section{CONCLUSIONS}

Particle creation at the stagnation surface of Poyntingdominated jets presents a natural mechanism for filling black hole jets with nonthermal particles near the horizon, as required by recent mm-VLBI observations of M87. This model provides an excellent quantitative description of a polar electron-positron cascade that can fill black hole jets with relativistically-hot plasma with sufficient abundance to explain the observed jet emission in M87.

Within the stagnation surface of astrophysical jets, large electric fields are anticipated, arising due to the charge starvation within the jet. These are capable of accelerating stray leptons to extremely high Lorentz factors, limited by inverseCompton cooling on the ambient soft-photon background, due to local synchrotron emission within the jet or through their production in an accretion disk. The up-scattered gamma rays are sufficiently energetic to initiate a pair catastrophe that ultimately self-consistently determines the structure and content of the jet near the stagnation surface, i.e., within the gap, beyond which sufficient charge densities exist to screen the accelerating electric fields. Typical gap thicknesses are much smaller than the gravitational radius, and thus largely independent of the global jet structure.

Subsequent to exiting the gap, the leptons typically have very large Lorentz factors, and thus participate in a post-gap inverseCompton cascade. While this does not increase the charge density, and therefore does not affect the jet structure, it does significantly increase the lepton density and decrease their specific energy. Typical lepton density enhancements can reach $10^{3}$, and thus the post-gap cascade can have a dramatic impact upon the particle content of astrophysical jets. The resulting pairs are then natural candidates for the relativistic lepton populations that produce the synchrotron emission in the cores of radio AGN. In particular, particle creation at the stagnation surface provides an excellent quantitative explanation of the horizon-scale emission observed in M87. In contrast, it vastly under produces the lepton content of Sgr A*'s emission region, consistent with the lack of an obvious jet in that source.

Gamma-ray luminosity produced by the stagnation surface pair cascade considerably exceeds that detected by Fermi/LAT for M87, though it is similar to those found for gamma-ray bright blazars. However, this naturally results from the strong beaming along the jet of the up-scattered emission, implying a viewing inclination larger than $\approx 9^{\circ}$. Thus, within the context of the stagnation surface pair catastrophe model we present here, the lack of bright high-energy gamma-ray emission from M87 favors the larger inclination implied by high-frequency radio observations. Nevertheless, future work should compute the angular dependence of the emergent inverse-Compton spectra and the potentially strong constraints on jet orientation that may result.

The authors thank Dimitrios Giannios, Ramesh Narayan and Alexander Philippov for helpful discussions. The authors would also like to thank the anonymous referee for many help- 
ful suggestions that have resulted in a much improved presentation. A.E.B. receives financial support from Perimeter Institute for Theoretical Physics and the Natural Sciences and Engineering Research Council of Canada through a Discovery Grant. A.T. was supported by a Princeton Center for Theoretical Science Fellowship and by NASA through the Einstein Fellowship Program, grant PF3-140131. The simulations presented in this article used computational resources supported by XSEDE allocation TG-AST100040 on NICS Kraken and Nautilus and TACC Lonestar, Longhorn, Ranch, and Stampede. A.T. thanks Perimeter Institute for hospitality and financial support of three visits to Perimeter Institute during which most of the work on this project was carried out.

\section{APPENDIX}

\section{A. RELATIVISTIC COUNTER-STREAMING PAIR TWO-STREAM INSTABILITY}

One might be concerned with the potential for heating of the counter-streaming electron and positron beams, ultimately radiated away as synchrotron emission. Here we estimate the growth rate of this variant of the classic two-stream instability. Note that in this case we really do have a one-dimensional problem; particles are strongly confined to field lines and thus can only participate in collective motions in the direction of motion.

The electrons and positron motions are governed by the Boltzmann equation, which upon some simplification gives the following relation for the charge perturbations in terms of the unperturbed lepton distribution functions (see, for example, the appendix of Broderick et al. 2012):

$$
\begin{aligned}
\rho_{1} & =\frac{i e^{2}}{m} \mathbf{E} \cdot \int\left(f_{0}^{+}+f_{0}^{-}\right) \frac{\mathbf{k}-\mathbf{k} \cdot \mathbf{v v}}{\gamma(\omega-\mathbf{k} \cdot \mathbf{v})^{2}} d^{3} p \\
& =\frac{i e^{2}}{m} E k \cdot \int \frac{f_{0}^{+}+f_{0}^{-}}{\gamma^{3}(\omega-k v)^{2}} d^{3} p,
\end{aligned}
$$

where in the second expression we have assumed that all the vectors are co-linear due to the afore mentioned dimensionality of the problem. Inserting $f_{0}^{ \pm}=(n / 2) \delta^{3}\left(\mathbf{p} \pm \mathbf{p}_{\mathbf{0}}\right)$ and performing the trivial integrals results in

$$
\rho_{1}=\frac{i e^{2} n}{2 m \gamma^{3}} E k\left[\frac{1}{(\omega-k v)^{2}}+\frac{1}{(\omega+k v)^{2}}\right] .
$$

From Gauss law $(\boldsymbol{\nabla} \cdot \mathbf{E}=4 \pi \rho)$ we then find

$$
i k E=4 \pi \rho_{1}=\frac{4 \pi i e^{2} n}{m \gamma^{3}} \frac{E k}{2}\left[\frac{1}{(\omega-k v)^{2}}+\frac{1}{(\omega+k v)^{2}}\right]
$$

or

$$
1-\frac{\omega_{P}^{2}}{2 \gamma^{3}}\left[\frac{1}{(\omega-k v)^{2}}+\frac{1}{(\omega+k v)^{2}}\right]=1-\frac{\omega_{P}^{2}}{2 \gamma^{3}} \frac{\omega^{2}+(k v)^{2}}{\left[\omega^{2}-(k v)^{2}\right]^{2}}=0
$$

or, with $\bar{k}=k v \gamma^{3 / 2} / \omega_{P}$ and $\bar{\omega}=\omega \gamma^{3 / 2} / \omega_{P}$,

$$
\begin{aligned}
\bar{\omega}^{4}-\left(2 \bar{k}^{2}+1\right) \bar{\omega}^{2}-\bar{k}^{2}\left(1-\bar{k}^{2}\right) & =0 \\
\Rightarrow \quad \bar{\omega}^{2} & =\bar{k}^{2}+\frac{1}{2} \pm \sqrt{\left(\bar{k}^{2}+\frac{1}{2}\right)^{2}+\bar{k}^{2}\left(1-\bar{k}^{2}\right)} \\
& =\bar{k}^{2}+\frac{1}{2} \pm \sqrt{2 \bar{k}^{2}+\frac{1}{4}}
\end{aligned}
$$

This is minimized for the second root (the - sign), when

$$
1-\frac{1}{\sqrt{2 \bar{k}^{2}+1 / 4}}=0 \Rightarrow \bar{k}^{2}=\frac{3}{8} \Rightarrow \bar{\omega}^{2}=-\frac{1}{8} \text {. }
$$

Thus, the growth rate of the relativistically counter-streaming pair instability is,

$$
\Gamma_{\text {CSI }}=\Im(\omega)=\frac{\omega_{P}}{\sqrt{8 \gamma^{3}}} .
$$

Within the gap the density given in Equation (18) sets a characteristic upper limit,

$$
n_{\mathrm{g}}=\frac{\nabla \cdot \mathbf{E}}{4 \pi e} \approx \frac{E}{4 \pi e \Delta}=3.3 R_{15}^{3 / 2} \Omega_{F,-4}^{3 / 2} B_{2}^{3 / 2} u_{s, 0}^{1 / 2} \eta_{\mathrm{th}}^{-1 / 2} \mathrm{~cm}^{-3} .
$$

Note that here the characteristic length scale is $\Delta$, i.e., much smaller than the distance to the stagnation surface, $10 r_{g}$. The corresponding plasma frequency is then

$$
\omega_{P} \approx 1.0 \times 10^{5} R_{15}^{3 / 4} \Omega_{F,-4}^{3 / 4} B_{2}^{3 / 4} u_{s, 0}^{1 / 4} \eta_{\mathrm{th}}^{-1 / 4} \mathrm{~Hz}
$$

and thus setting $\gamma=\gamma_{\max }$,

$$
\Gamma_{\mathrm{CSI}} \approx 4.2 \times 10^{-9} u_{s, 0} \eta_{\mathrm{th}}^{-1 / 4} \mathrm{~Hz},
$$

which is typically exceedingly slow in comparison to the lightcrossing time of the gap. The corresponding cooling length is $\ell_{\mathrm{CSI}} \approx c / \Gamma_{\mathrm{CSI}}$.

\section{B. ONE-DIMENSIONAL, APPROXIMATE GAP STRUCTURE}

The gap structure is set by the competition between pair production and the screening of the externally applied electric field by the resulting charge separation. In principle, this requires a solution to the fully non-linear coupled Maxwell-Boltzmann equations, including inverse Compton scattering. Particle-incell (PIC) simulations could be one such approach. However, in practice, a rough estimate of the gap structure may be obtained via an approximate form of the Maxwell-Boltzmann equations, in which the non-linearity is manifested by boundary conditions.

Here we explicitly construct the solutions to the simple gap particle population model listed in Section 2.2. We assume that the electric field within the gap $E_{g}$ is sufficient to instantaneously drive particles to their inverse Compton limited velocities, and thus the particle distributions are essentially monoenergetic. The resulting particle distribution will evolve to screen the externally applied $E_{g}$, preventing further acceleration and saturating the growth of the gap lepton density. Note that in this way the linear model encapsulates the nonlinearity inherent in the full Maxwell-Boltzmann equations.

For concreteness, we assume that the electric field is positive in the upward direction, corresponding to the direction of the guiding magnetic field line, in which case the electrons and positrons accelerate downward and upward, respectively. Then, the number densities of the pairs $\left(n_{e^{+}}, n_{e^{-}}\right)$and upward and downward propagating gamma-rays $\left(n_{\gamma^{+}}, n_{\gamma^{-}}\right)$evolve due to pair production and inverse Compton scattering via:

$$
\begin{aligned}
& \dot{n}_{e^{+}}+c \partial_{z} n_{e^{+}}=\alpha_{\gamma \gamma} c\left(n_{\gamma^{+}}+n_{\gamma^{-}}\right) \\
& \dot{n}_{e^{-}}-c \partial_{z} n_{e^{-}}=\alpha_{\gamma \gamma} c\left(n_{\gamma^{+}}+n_{\gamma^{-}}\right) \\
& \dot{n}_{\gamma^{+}}+c \partial_{z} n_{\gamma^{+}}=\alpha_{\mathrm{IC}} c n_{e^{+}}-\alpha_{\gamma \gamma} c n_{\gamma^{+}} \\
& \dot{n}_{\gamma^{-}}-c \partial_{z} n_{\gamma^{-}}=\alpha_{\mathrm{IC}} c n_{e^{-}}-\alpha_{\gamma \gamma} c n_{\gamma^{-}} .
\end{aligned}
$$

where $\alpha_{\gamma \gamma} \equiv \sigma_{\gamma \gamma} n_{s}, \alpha_{\mathrm{IC}} \equiv \sigma_{T} n_{s}$, and $n_{s}$ is the number density of seed photons. Pairs are generated by both upward and 
downward propagating photons, though as assumed travel only in their prescribed directions. Thus, the upward (downward) propagating gamma-rays are produced by inverse Compton scattering by only the positrons (electrons).

While Equation (B1) is manifestly one-dimensional, the stagnation surface itself extends transversely over a macroscopic scales, i.e., typically much longer than $\ell_{I C}$ or $\ell_{\gamma \gamma}$. The strong beaming of the up-scattered gamma rays by the highly relativistic, accelerated leptons, implies that regions that are separated by a transverse distance of more $\Delta / \gamma$, where $\Delta$ is the gap height to be determined below, execute independent pair catastrophes. As a result, the stagnation surface is comprised of a large number $\left(\approx \gamma^{2} R^{2} / \Delta^{2}\right)$ of essentially independently evolving one-dimensional, pair cascades, each described by Equation (B1).

The pair cascade is inherently unstable, as will be shown in detail in Section B.2 though is clear from the nature of the quenching: pairs form and accelerate until the resulting charge segregation screens the externally applied electric field. As a consequence, the microscopic gap structure, i.e., that within a single causally connected tube, is highly variable. However, the macroscopic gap structure, comprised by very many causally connected tubes, is well described by an ensemble average over microscopic tubes at different stages of the discharge cycle, i.e., the time-averaged structure of a single microscopic region. Due to the linearity of Equation (B1), this corresponds immediately to the stationary solution, which we address first.

\section{B.1. Stationary Structure}

Assuming stationarity, all the time derivatives vanish, and Equation (B1) is a set of coupled ordinary differential equations for the gap structure:

$$
\begin{aligned}
\partial_{z} n_{e^{+}} & =\alpha_{\gamma \gamma}\left(n_{\gamma^{+}}+n_{\gamma^{-}}\right) \\
-\partial_{z} n_{e^{-}} & =\alpha_{\gamma \gamma}\left(n_{\gamma^{+}}+n_{\gamma^{-}}\right) \\
\partial_{z} n_{\gamma^{+}} & =\alpha_{\mathrm{IC}} n_{e^{+}}-\alpha_{\gamma \gamma} n_{\gamma^{+}} \\
-\partial_{z} n_{\gamma^{-}} & =\alpha_{\mathrm{IC}} n_{e^{-}}-\alpha_{\gamma \gamma} n_{\gamma^{-}} .
\end{aligned}
$$

Choosing $z=0$ to lie in the gap center, symmetry requires that

$$
n_{e^{+}}(z)=n_{e^{-}}(-z) \equiv f(z)
$$

and similarly

$$
n_{\gamma^{+}}(z)=n_{\gamma^{-}}(-z) \equiv g(z)
$$

Thus, Equations (B2) reduce to

$$
f^{\prime}=\alpha_{\gamma \gamma}[g(z)+g(-z)]
$$

and

$$
g^{\prime}=\alpha_{\mathrm{IC}} f-\alpha_{\gamma \gamma} g,
$$

where for compactness we have denoted $\partial_{z}$ with primes. Taking the second derivative of the second equation and inserting the first, we obtain the following for $g$ :

$$
g^{\prime \prime}+\alpha_{\gamma \gamma} g^{\prime}-\alpha_{\mathrm{IC}} \alpha_{\gamma \gamma}[g(z)+g(-z)]=0 .
$$

Noting that $g^{\prime}(-z)=-[g(-z)]^{\prime}$ and $g^{\prime \prime}(-z)=[g(-z)]^{\prime \prime}$, this implies

$$
\begin{gathered}
{[g(z)+g(-z)]^{\prime \prime}-2 \alpha_{\mathrm{IC}} \alpha_{\gamma \gamma}[g(z)+g(-z)]=0} \\
\alpha_{\gamma \gamma}[g(z)-g(-z)]^{\prime}=0,
\end{gathered}
$$

from which, with the required symmetry, we obtain the solutions

$$
g(z)=A \cosh \left(\sqrt{2 \alpha_{\mathrm{IC}} \alpha_{\gamma \gamma}} z\right) .
$$

We may now construct $f$ by integration:

$$
f=A \sqrt{\frac{\alpha_{\gamma \gamma}}{2 \alpha_{\mathrm{IC}}}} \sinh \left(\sqrt{2 \alpha_{\mathrm{IC}} \alpha_{\gamma \gamma} z}\right)+B .
$$

The contribution to the electric field from the pairs within the gap may be obtained directly by integrating Gauss' law,

$$
\begin{aligned}
E_{\|}^{\prime} & =4 \pi e\left(n_{e^{+}}-n_{e^{-}}\right)=4 \pi e[f(z)-f(-z)] \\
\Rightarrow \quad E_{\|} & =\frac{4 \pi e A}{\alpha_{\mathrm{IC}}} \cosh \left(\sqrt{2 \alpha_{\mathrm{IC}} \alpha_{\gamma \gamma} z}\right)+C .
\end{aligned}
$$

When $A=0$, i.e., the charge density vanishes, the total electric field is simply that due to the gap, $E_{g}$. The particle cascade saturates when the gap is closed, i.e., $E_{\|} \approx 0$ at the center, and $E_{\|}=E_{g}$ at the gap boundary, where it is presumably screened by MHD processes, taken to lie at a height $z_{g}$. The first condition gives

$$
C=-\frac{4 \pi e A}{\alpha_{\mathrm{IC}}} .
$$

The second then sets $A$ in terms of the gap size:

$$
A=\frac{\alpha_{\mathrm{IC}} E_{g}}{4 \pi e(\cosh \delta-1)},
$$

where $\delta \equiv \sqrt{2 \alpha_{\mathrm{IC}} \alpha_{\gamma \gamma}} z_{g}=2 z_{g} / \Delta$, where $\Delta \equiv \sqrt{2 \ell_{\mathrm{IC}} \ell_{\gamma \gamma}}$ is the characteristic gap length scale.

Because all particle production is assumed to occur within the gap, only outgoing particles exist above and below the gap boundaries. The condition that $f \geq 0$ everywhere within the gap then requires that the density of each species vanishes at a corresponding boundary, set by their respective directions of motion, i.e., the positron and electron densities vanishes at the bottom and top of the gap, respectively. This implies,

$$
B=A \sqrt{\frac{\alpha_{\gamma \gamma}}{2 \alpha_{\mathrm{IC}}}} \sinh \delta,
$$

from which we infer the particle densities at the top of the gap,

$$
n_{e^{+}}\left(z_{g}\right) \approx \frac{\sqrt{2 \alpha_{\mathrm{IC}} \alpha_{\gamma \gamma}} E_{g}}{4 \pi e} \frac{\sinh \delta}{\cosh \delta-1} .
$$

It is clear from the above that the natural gap scale is $\Delta$. However, the density at the top of the gap is a weak function of $\delta$, with $\sinh \delta /(\cosh \delta-1)$ dropping to 2 at $\delta \simeq 1$ and falling below 1.3 for $\delta>2$. Thus, independent of the precise gap thickness, the density at the top of the gap is

$$
n_{e^{+}} \approx 2 \frac{E_{g}}{4 \pi e \Delta}
$$

which is directly comparable to Equation (18). Kinetic plasma simulations are desirable to verify this linearized model but are beyond the scope of this work.

Equation (B16) gives a value of charge density that is a factor $R / \Delta=10 r_{g} / \Delta \sim 10^{5}$ times greater than the Goldreich-Julian density, $\rho_{\mathrm{GJ}} \sim \Omega B / 2 \pi c$. Consequently, the gap structure implies a large pair multiplicity that is, however, not unusual for magnetospheric cascades, e.g., in the context of pulsars (TA13). In fact, the multiplicity $\eta=R / \Delta$ implied by Equation (B16) is in good agreement with the simulation results of TA13 (see the discussion after Equation 18). 


\section{B.2. Gap Dynamics}

The gap model described above is already linearized, simplifying the discussion of its dynamics considerably. Here we look at small, harmonic perturbations about the steady state configuration. We have some freedom to measure time and distance in convenient units, which to simplify the following expressions, we do in $\ell_{\gamma \gamma}=1 / \alpha_{\gamma \gamma} c$. Then, defining $\varpi \equiv \omega / \alpha_{\gamma \gamma} c$, $\kappa \equiv k / \alpha_{\gamma \gamma} c$, and $\zeta \equiv \alpha_{\mathrm{IC}} / \alpha_{\gamma \gamma}$, the equation for the perturbations becomes

$$
\begin{aligned}
-i(\varpi-\kappa) \delta n_{e^{+}} & =\delta n_{\gamma^{+}}+\delta n_{\gamma^{-}} \\
-i(\varpi+\kappa) \delta n_{e^{+}} & =\delta n_{\gamma^{+}}+\delta n_{\gamma^{-}} \\
-i(\varpi-\kappa) \delta n_{\gamma^{+}} & =\zeta \delta n_{e^{+}}-\delta n_{\gamma^{+}} \\
-i(\varpi+\kappa) \delta n_{\gamma^{-}} & =\zeta \delta n_{e^{-}}-\delta n_{\gamma^{-}} .
\end{aligned}
$$

This may be trivially rearranged to define the modes as a standard eigenmode problem:

$$
\left(\begin{array}{cccc}
\kappa & 0 & i & i \\
0 & -\kappa & i & i \\
i \zeta & 0 & \kappa-i & 0 \\
0 & i \zeta & 0 & -\kappa-i
\end{array}\right)\left(\begin{array}{l}
\delta n_{e^{+}} \\
\delta n_{e^{-}} \\
\delta n_{\gamma^{+}} \\
\delta n_{\gamma^{-}}
\end{array}\right)=\varpi\left(\begin{array}{l}
\delta n_{e^{+}} \\
\delta n_{e^{-}} \\
\delta n_{\gamma^{+}} \\
\delta n_{\gamma^{-}}
\end{array}\right)
$$

The associated four mode-specific dispersion relations are

$$
\varpi=-\frac{i}{2}\left[1 \pm \sqrt{1+4 \zeta-4 \kappa^{2} \pm 4 \sqrt{\zeta^{2}-(1+4 \zeta) \kappa^{2}}}\right],
$$

where every combination of the two \pm may be chosen. Two of the four modes are unstable (-- and -+$)$, both of which correspond to the pair catastrophe (related to the symmetric and anti-symmetric components). The maximum growth rate is

$$
\left.\Gamma_{--}\right|_{\kappa=0} \equiv \alpha_{\gamma \gamma} c \Im\left(\bar{\omega}_{--}\right)=\frac{\sqrt{\alpha_{\gamma \gamma}^{2}+8 \alpha_{\gamma \gamma} \alpha_{\mathrm{IC}}}-\alpha_{\gamma \gamma}}{2} c .
$$

Thus, the growth time, $\Gamma_{--}^{-1}$, is roughly the light crossing time of the open gap. The physical origin of the instability is easy to understand: additional leptons induce additional inverse Compton scattered photons which, in turn, produces additional electrons, causing the process to exponentiate.

\section{DIRECT INVERSE COMPTON FLUX DENSITY ESTIMATES}

Here we construct a simple model with which to estimate the direct inverse Compton signal from the gap-accelerated leptons. The critical input assumption is that the leptons evolve solely due to inverse Compton cooling after leaving the gap. Simplifying assumptions, that certainly hold in the case of M87 and are likely to hold generically, include a broken power-law distribution for the seed photons and a uniform seed photon density over the cooling length scales of interest. As in section 3.2, for compactness we will measure energies in units of electron rest mass.

For concreteness, the seed photon distribution is take to be of the form given in Equation (30)

$$
\frac{d n_{s}}{d \epsilon_{s}}=\frac{u_{s}}{\epsilon_{b}^{2}} \frac{(\alpha-1)(1-\beta)}{\alpha-\beta} \begin{cases}\left(\epsilon_{s} / \epsilon_{b}\right)^{-(\beta+1)} & \epsilon_{s} \leq \epsilon_{b} \\ \left(\epsilon_{s} / \epsilon_{b}\right)^{-(\alpha+1)} & \epsilon_{s}>\epsilon_{b},\end{cases}
$$

i.e., a broken power-law with a spectral indexes of $\alpha$ and $\beta$ above and below the break, respectively. The above is normalized such that the seed photon energy density is $u_{s}$.
The post-gap lepton population has a height-dependent, mono-energetic distribution:

$$
\frac{d n}{d \gamma}=n_{\infty} \delta[\gamma-\gamma(z)]
$$

where $\gamma(z)$ is set by inverse Compton cooling, and determined by

$$
\frac{d \gamma}{d z}=-\frac{4 \sigma_{T} u_{s}}{3 m c^{2}} \gamma^{2}
$$

subject to the initial condition that at the top of the gap, $\gamma=\gamma_{\infty}$. While this may be trivially integrated, only the above expression is required below.

Given a mono-energetic electron distribution, the energies of the up-scattered gamma ray and the initial seed photon are related by

$$
\epsilon_{s}=\frac{\epsilon}{2 \gamma(\gamma-\epsilon)} \Rightarrow \frac{d \epsilon_{s}}{d \epsilon}=\frac{1}{2(\gamma-\epsilon)^{2}},
$$

where we have expressed these in a slightly different form than usual, obtaining the seed photon energy in terms of the gammaray energy.

The resulting gamma-ray flux is then

$$
F_{\epsilon}=\int d \gamma d z \sigma_{T} c \frac{d n}{d \gamma} \frac{d n_{s}}{d \epsilon_{s}} \frac{d \epsilon_{s}}{d \epsilon} \epsilon \frac{r^{2}}{D^{2}},
$$

where the factor of $d \epsilon_{s} / d \epsilon$ simply converts from per unit seed photon energy to per unit gamma-ray energy. The beaming corrections (enhanced emission along the direction of the electron momentum and reduced viewing area) cancel as described in the main text. Affecting the integral over $\gamma$ is trivial due to the mono-energetic nature of the electron distribution, hence,

$$
\begin{aligned}
F_{\epsilon}= & \sigma_{T} c n_{\infty} \epsilon \frac{r^{2}}{D^{2}} \int_{z_{\text {gap }}}^{\infty} d z \frac{d n_{s}}{d \epsilon_{s}} \frac{1}{2[\gamma(z)-\epsilon]^{2}} \\
= & \sigma_{T} c n_{\infty} \epsilon \frac{r^{2}}{D^{2}} \int_{\gamma_{\infty}}^{1} d \gamma \frac{d n_{s} / d \epsilon_{s}}{d \gamma / d z} \frac{1}{2(\gamma-\epsilon)^{2}} \\
= & \frac{3 m c^{3} n_{\infty}}{\epsilon_{b}} \frac{(\alpha-1)(1-\beta)}{\alpha-\beta} \frac{r^{2}}{D^{2}} \\
& \times\left[\left(\frac{\epsilon}{2 \epsilon_{b}}\right)^{-\beta} \int_{\gamma_{b}}^{\gamma_{\infty}} d \gamma[\gamma(\gamma-\epsilon)]^{\beta-1}\right. \\
& \left.+\left(\frac{\epsilon}{2 \epsilon_{b}}\right)^{-\alpha} \int_{1}^{\gamma_{b}} d \gamma[\gamma(\gamma-\epsilon)]^{\alpha-1}\right],
\end{aligned}
$$

where

$$
\gamma_{b}=\frac{\epsilon}{2}\left(1+\sqrt{1+\frac{2}{\epsilon \epsilon_{b}}}\right) \approx \sqrt{\frac{\epsilon}{2 \epsilon_{b}}},
$$

is the electron Lorentz factor that up scatters seed photons at the break to the desired observed energy. For the cases of interest, $\epsilon \ll 1 / \epsilon_{b}$, and the term in the radical dominates as shown. In the limit that $\epsilon \ll \gamma_{b}$, the remaining integrals may then be approximated by

$$
\begin{aligned}
\int_{\sqrt{\epsilon / 2 \epsilon_{b}}}^{\gamma_{\infty}} d \gamma[\gamma(\gamma-\epsilon)]^{\beta-1} & \left.\approx \frac{\gamma^{2 \beta-1}}{2 \beta-1}\right|_{\sqrt{\epsilon / 2 \epsilon_{b}}} ^{\gamma_{\infty}} \\
& \approx \frac{1}{1-2 \beta}\left(\frac{\epsilon}{2 \epsilon_{b}}\right)^{\beta-1 / 2}
\end{aligned}
$$


where we have assumed that $\beta<1 / 2$, and

$$
\int_{\epsilon}^{\sqrt{\epsilon / 2 \epsilon_{b}}} d \gamma[\gamma(\gamma-\epsilon)]^{\alpha-1} \approx \frac{1}{2 \alpha-1}\left(\frac{\epsilon}{2 \epsilon_{b}}\right)^{\alpha-1 / 2}
$$

Thus,

$$
F_{\epsilon}=\frac{6 m^{2} c^{5} n_{\infty}}{\epsilon_{b}} \frac{(\alpha-1)(1-\beta)}{(2 \alpha-1)(1-2 \beta)} \frac{r^{2}}{D^{2}}\left(\frac{\epsilon}{2 \epsilon_{b}}\right)^{-1 / 2}
$$

where in the final expression the factors of $m c^{2}$ have been reintroduced. The typical flux at energy $\epsilon$ is then

$$
\begin{aligned}
\epsilon F_{\epsilon} & =\int_{\epsilon}^{\infty} d \epsilon F_{\epsilon} \\
& \approx 12 m^{2} c^{5} n_{\infty} \frac{(\alpha-1)(1-\beta)}{(2 \alpha-1)(1-2 \beta)} \frac{r^{2}}{D^{2}}\left(\frac{\epsilon}{2 \epsilon_{b}}\right)^{1 / 2} \\
& \approx 12 \gamma_{b} m c^{3} n_{\infty} \frac{(\alpha-1)(1-\beta)}{(2 \alpha-1)(1-2 \beta)} \frac{r^{2}}{D^{2}}
\end{aligned}
$$

\section{REFERENCES}

Ackermann, M., et al. 2011, ApJ, 743, 171

—. 2013, ArXiv:1306.6772

Agol, E. 2000, ApJ, 538, L121

Baath, L. B., et al. 1992, A\&A, 257, 31

Baes, M., et al. 2010, A\&A, 518, L53

Beskin, V. S., Istomin, Y. N., \& Parev, V. I. 1992, Soviet Ast., 36, 642

Biretta, J. A., Junor, W., \& Livio, M. 2002, New Astronomy Reviews, 46, 239

Blandford, R. D., \& Znajek, R. L. 1977, MNRAS, 179, 433

Broderick, A. E., \& Loeb, A. 2006, ApJ, 636, L109

-. 2009, ApJ, 697, 1164

Broderick, A. E., Narayan, R., Kormendy, J., Perlman, E. S., Rieke, M. J., \&

Doeleman, S. S. 2015, ApJ, 805, 179

de Gasperin, F., et al. 2012, A\&A, 547, A56

Dexter, J., McKinney, J. C., \& Agol, E. 2012, MNRAS, 421, 1517

Doeleman, S. S., et al. 2012, Science, 338, 355

Falcke, H., \& Markoff, S. 2000, A\&A, 362, 113

Gebhardt, K., Adams, J., Richstone, D., Lauer, T. R., Faber, S. M., Gültekin,

K., Murphy, J., \& Tremaine, S. 2011, ApJ, 729, 119

Ghez, A. M., et al. 2008, ApJ, 689, 1044

Gillessen, S., Eisenhauer, F., Trippe, S., Alexander, T., Genzel, R., Martins, F., \& Ott, T. 2009, ApJ, 692, 1075

Gould, R. J., \& Schréder, G. P. 1967, Physical Review, 155, 1404

Hirotani, K., \& Okamoto, I. 1998, ApJ, 497, 563

Junor, W., \& Biretta, J. A. 1995, AJ, 109, 500
Komissarov, S. S. 2004, MNRAS, 350, 1431

Kovalev, Y. Y., et al. 2005, AJ, 130, 2473

Lasota, J.-P., Gourgoulhon, E., Abramowicz, M., Tchekhovskoy, A., \& Narayan, R. 2014, Phys. Rev. D, 89, 024041

Lee, S.-S., Lobanov, A. P., Krichbaum, T. P., Witzel, A., Zensus, A., Bremer, M., Greve, A., \& Grewing, M. 2008, AJ, 136, 159

Levinson, A., \& Eichler, D. 2003, ApJ, 594, L19

Levinson, A., \& Rieger, F. 2011, ApJ, 730, 123

Li, Z., Morris, M. R., \& Baganoff, F. K. 2013, ArXiv e-prints

Lister, M. L., \& Homan, D. C. 2005, AJ, 130, 1389

Lonsdale, C. J., Doeleman, S. S., \& Phillips, R. B. 1998, AJ, 116, 8

Ly, C., Walker, R. C., \& Wrobel, J. M. 2004, AJ, 127, 119

Marrone, D. P. 2006, PhD thesis, Harvard University

Marrone, D. P., Moran, J. M., Zhao, J.-H., \& Rao, R. 2007, ApJ, 654, L57

McKinney, J. C., Tchekhovskoy, A., \& Blandford, R. D. 2012, MNRAS, 423, 3083

Mościbrodzka, M., \& Falcke, H. 2013, A\&A, 559, L3

Mościbrodzka, M., Gammie, C. F., Dolence, J. C., \& Shiokawa, H. 2011, ApJ, 735,9

Mościbrodzka, M., Gammie, C. F., Dolence, J. C., Shiokawa, H., \& Leung, P. K. 2009, ApJ, 706, 497

Narayan, R., \& Yi, I. 1994, ApJ, 428, L13

-. 1995, ApJ, 452, 710

Neronov, A., \& Aharonian, F. A. 2007, ApJ, 671, 85

Owen, F. N., Eilek, J. A., \& Kassim, N. E. 2000, ApJ, 543, 611

Penna, R. F., Narayan, R., \& Sa̧dowski, A. 2013, MNRAS, 436, 3741

Perlman, E. S., et al. 2007, ApJ, 663, 808

Petrov, L., Hirota, T., Honma, M., Shibata, K. M., Jike, T., \& Kobayashi, H. 2007, AJ, 133, 2487

Shcherbakov, R. V., Penna, R. F., \& McKinney, J. C. 2012, ApJ, 755, 133

Sparks, W. B., Biretta, J. A., \& Macchetto, F. 1996, ApJ, 473, 254

Spencer, R. E., \& Junor, W. 1986, Nature, 321, 753

Stawarz, Ł., Aharonian, F., Kataoka, J., Ostrowski, M., Siemiginowska, A., \& Sikora, M. 2006, MNRAS, 370, 981

Su, M., \& Finkbeiner, D. P. 2012, ApJ, 753, 61

Su, M., Slatyer, T. R., \& Finkbeiner, D. P. 2010, ApJ, 724, 1044

Tchekhovskoy, A. 2015, in Astrophysics and Space Science Library, Vol. 414, Astrophysics and Space Science Library, ed. I. Contopoulos, D. Gabuzda, \& N. Kylafis, 45

Tchekhovskoy, A., \& McKinney, J. C. 2012, MNRAS, 423, L55

Tchekhovskoy, A., McKinney, J. C., \& Narayan, R. 2012, Journal of Physics Conference Series, 372, 012040

Tchekhovskoy, A., Narayan, R., \& McKinney, J. C. 2010, ApJ, 711, 50

-. 2011, MNRAS, 418, L79

Timokhin, A. N., \& Arons, J. 2013, MNRAS, 429, 20

Vincent, S., \& Lebohec, S. 2010, MNRAS, 409, 1183

Walker, R. C., Ly, C., Junor, W., \& Hardee, P. J. 2008, Journal of Physics Conference Series, 131, 012053

Walsh, J. L., Barth, A. J., Ho, L. C., \& Sarzi, M. 2013, ApJ, 770, 86

Yuan, F., Markoff, S., \& Falcke, H. 2002, A\&A, 383, 854

Yuan, F., Quataert, E., \& Narayan, R. 2003, ApJ, 598, 301

-. 2004, ApJ, 606, 894 